\title{
1. Dual Role of the Active Site Residues of Thermus thermophilus 3-Isopropylmalate Dehydrogenase: Chemical Catalysis and Domain ${ }_{3}$ Closure
}

${ }_{4}$ Éva Gráczer, ${ }^{\dagger}$ Tamás Szimler, ${ }^{\dagger}$ Anita Garamszegi, ${ }^{\dagger}$ Petr V. Konarev, ${ }^{\ddagger}, \#$ Anikó Lábas, ${ }^{\S}$ Julianna Oláh, ${ }^{*}, \S$ ${ }_{s}$ Anna Palló,,$"$ Dmitri I. Svergun, ${ }^{\ddagger}$ Angelo Merli, ${ }^{\perp}$ Péter Závodszky, ${ }^{\dagger}$ Manfred S. Weiss, ${ }^{\circledR}$ and Mária Vas* ${ }^{* \dagger}$

$6{ }^{\dagger}$ Institute of Enzymology, Research Centre for Natural Sciences, Hungarian Academy of Sciences, Magyar tudósok krt. 2., H-1117

7 Budapest, Hungary

8 European Molecular Biology Laboratory, Hamburg Outstation, Notkestrasse 85, 22603 Hamburg, Germany

$9{ }^{\S}$ Department of Inorganic and Analytical Chemistry, Budapest University of Technology and Economics, Gellért tér 4., H-1111

10 Budapest, Hungary

11 "Institute of Organic Chemistry, Research Centre for Natural Sciences, Hungarian Academy of Sciences, Magyar tudósok krt. 2.,

$12 \mathrm{H}-1117$ Budapest, Hungary

${ }_{13}{ }^{\perp}$ Dipartimento di Bioscienze, Universitá degli Studi di Parma, Viale G.P. Usberti 23/A, I-43100 Parma, Italy

${ }_{14}{ }^{\circledR}$ Macromolecular Crystallography (HZB-MX), Helmholtz-Zentrum Berlin für Materialien und Energie, Albert-Einstein-Strasse 15, 15 D-12489 Berlin, Germany

16 S Supporting Information

17 ABSTRACT: The key active site residues K185, Y139, D217,

18 D241, D245, and N102 of Thermus thermophilus 3-

19 isopropylmalate dehydrogenase (Tt-IPMDH) have been

20 replaced, one by one, with Ala. A drastic decrease in the $k_{\text {cat }}$

21 value ( $0.06 \%$ compared to that of the wild-type enzyme) has

22 been observed for the K185A and D241A mutants. Similarly,

23 the catalytic interactions ( $K_{\mathrm{m}}$ values) of these two mutants

24 with the substrate IPM are weakened by more than 1 order of

25 magnitude. The other mutants retained some $(1-13 \%)$ of the

Activity $\left(k_{\text {cat }}, \min ^{-1}\right)$ of IPMDH

\begin{tabular}{lc} 
Wild-type & $238+/-30$ \\
$\frac{\text { K185A }}{\text { D241A }}$ & $0.15+/-0.05$ \\
\hline D217A & $0.15+/-0.05$ \\
& $2.7+/-0.5$
\end{tabular}

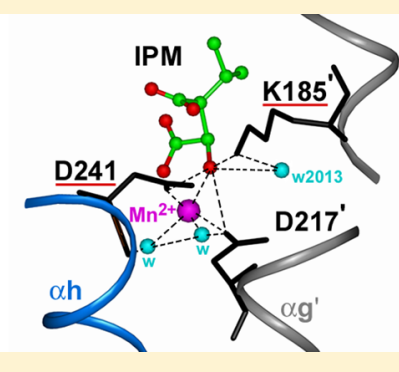
catalytic activity of the wild-type enzyme and do not exhibit appreciable changes in the substrate $K_{\mathrm{m}}$ values. The $\mathrm{pH}$ dependence of the wild-type enzyme activity $(\mathrm{pK}=7.4)$ is shifted toward higher values for mutants $\mathrm{K} 185 \mathrm{~A}$ and $\mathrm{D} 241 \mathrm{~A}$ ( $\mathrm{pK}$ values of 8.4 and 8.5, respectively). For the other mutants, smaller changes have been observed. Consequently, K185 and D241 may constitute a proton relay system that can assist in the abstraction of a proton from the $\mathrm{OH}$ group of IPM during catalysis. Molecular dynamics simulations provide strong support for the neutral character of K185 in the resting state of the enzyme, which implies that K185 abstracts the proton from the substrate and D241 assists the process via electrostatic interactions with K185. Quantum mechanics/molecular mechanics calculations revealed a significant increase in the activation energy of the hydride transfer of the redox step for both D217A and D241A mutants. Crystal structure analysis of the molecular contacts of the investigated residues in the enzyme-substrate complex revealed their additional importance (in particular that of K185, D217, and D241) in stabilizing the domain-closed active conformation. In accordance with this, small-angle X-ray scattering measurements indicated the complete absence of domain closure in the cases of D217A and D241A mutants, while only partial domain closure could be detected for the other mutants. This suggests that the same residues that are important for catalysis are also essential for inducing domain closure.

39 3-Isopropylmalate dehydrogenase (IPMDH) is a member of 40 the $\beta$-hydroxyacid oxidative decarboxylase family, to which also 41 isocitrate dehydrogenase, homoisocitrate dehydrogenase, tarta42 rate dehydrogenase, and malic enzyme belong (cf. ref 1 for a 43 review). Evidence from structural, ${ }^{2-4}$ bioinformatics, ${ }^{1}$ and 44 biochemical studies (mutation analysis, $\mathrm{pH}$ profiles, etc. $)^{4-8}$ 45 suggests that the catalytic apparatus of these related enzymes 46 are similar (including the critical role of a Lys-Tyr pair), 47 although there may be some uncertainties concerning the role 48 of each amino acid side chain in the active site. In particular, contradictory conclusions were drawn about the contribution of 49 the active site aspartates in the catalysis by isocitrate ${ }_{50}$ dehydrogenase. As for IPMDH, functional studies based on 51 mutational analysis are rather scarce, ${ }^{10-12}$ and the role of the 52 active site Lys and Asp sid -chains has not yet been tested. 53

Received: July 24, 2015

Revised: December 10, 2015 
54 IPMDH catalyzes the oxidation and decarboxylation of $55(2 R, 3 S)$-3-isopropylmalate (IPM) to 2-oxo-4-methyl-penta56 noate (2-oxo-isocaproate) in the presence of $\mathrm{NAD}^{+}$and a 57 divalent cation $\left(\mathrm{Mn}^{2+}\right.$ or $\left.\mathrm{Mg}^{2+}\right)$ in the leucine biosynthetic 58 pathway of bacteria, fungi, and plants. The role of K185 of 59 Thermus thermophilus ( $T t$ ) IPMDH, a conserved active site 60 residue, as a catalytic base in aiding deprotonation of the $\mathrm{OH}$ 61 group of the substrate IPM has been recently proposed, on the 62 basis of evidence from the completely closed crystal structure of 63 the nonfunctioning $\mathrm{Tt}$-IPMDH$-\mathrm{Mn}^{2+}-\mathrm{IPM}-\mathrm{NADH}$ quater64 nary complex in combination with quantum mechanics/ 65 molecular mechanics (QM/MM) calculations. ${ }^{13}$ This notion 66 is in accordance with the previous suggestion of the catalytic 67 role of the invariant K230 of Escherichia coli isocitrate 68 dehydrogenase. ${ }^{4}$ In the work presented here, besides this Lys 69 (K185 in Tt-IPMDH), we directly investigate the roles of other 70 conserved active site residues (Y139, D217, D241, D245, and $71 \mathrm{~N} 102$ ) of Tt-IPMDH by site-directed mutagenesis. All these 72 residues, except N102, are in direct contact with the substrate. 73 The side chain of N102, however, was suggested to contribute 74 to the activity through regulating the communication of the two 75 domains. $^{14}$

76 Indeed, in addition to the possible participation of these 77 active site side chains in the chemical catalysis, a further exciting 78 aspect is their simultaneous involvement in the allosteric 79 regulation of domain closure that leads to the optimal 80 alignment of the reacting substrates. In general, there are 81 many examples of proteins/enzymes where domain movements 82 are evidenced as an integral part of their function (e.g., refs 83 15-25). In fact, mechanisms of domain movement are 84 considered to be among the most general problems of both 85 enzymology and protein chemistry: operation of hinge bending 86 or shearing motion of domains and the route of allosteric 87 propagation of the effects of substrates toward the hinges or 88 shearing surfaces by participating important conserved side 89 chains deserve wide interest. Hinges between the two domains 90 have been identified in cases of oxidative decarboxylases, such 91 as the malic enzyme, ${ }^{26}$ isocitrate dehydrogenase, ${ }^{4}$ tartrate 92 dehydrogenase, ${ }^{3}$ and IPMDH. ${ }^{14}$ However, an open question 93 discussed in several reviews is how the allosteric effects of the 94 bound substrates are propagated within a protein molecule 95 toward the molecular hinges. In other words, the structural 96 principles (definitive, longer/shorter allosteric pathways from 97 the substrate binding sites) that govern movement of hinges in 98 most cases have not been clarified.

99 In this work, besides identifying the important catalytic 100 residues of $\mathrm{Tt}$-IPMDH, we aimed to elaborate possible 101 conformational pathways through which their molecular 102 contacts may lead to domain closure. These suggested 103 mechanisms are also tested and confirmed by FRET and 104 SAXS measurements conducted with the respective mutants.

\section{MATERIALS AND METHODS}

106 Enzymes and Chemicals. The conserved active site 107 residues (K185, Y139, D217, D241, D245, and N102) of Tt$108 \mathrm{IPMDH}$ were mutated to Ala using the QuickChange site109 directed mutagenesis kit. The modified enzymes were ex110 pressed and purified using the previously published method 111 applied for the wild-type enzyme. ${ }^{30}(2 R, 3 S)$-3-Isopropylmalic 112 acid (IPM) was purchased from Wako Biochemicals (Japan), 113 and $\mathrm{NAD}^{+}$and $\mathrm{NADH}$ were from Sigma. All other chemicals 114 were commercially available high-purity grade products.
SDS-PAGE and Native Gel Electrophoresis. SDS gel 115 electrophoresis was conducted using the method of Laemmli. ${ }^{31} 116$ Native gel electrophoresis was performed using the method of 117 Ornstein. ${ }^{32}$ The resolving and stacking gels were 12.5 and 5\%, 118 respectively. IPMDH $(3 \mu \mathrm{g})$ was loaded into the gel, and the 119 electrophoresis was conducted at $180 \mathrm{~V}$ in a buffer of $\mathrm{pH} 8.3120$ for $1 \mathrm{~h}$. The gels were stained with Coomassie Brilliant Blue G- 121 250.

Far- and Near-UV CD Spectra. CD measurements were 123 performed with a Jasco J-720 spectropolarimeter equipped with 124 a Neslab RTE 111 computer-controlled thermostat. Far-UV 125 CD spectra were recorded in the range of 190-260 nm; the 126 cuvette with a $1 \mathrm{~mm}$ path length was used at a protein 127 concentration of $0.6 \mathrm{mg} / \mathrm{mL}$ ( $15 \mu \mathrm{M}$ monomer). For recording 128 near-UV CD spectra in the range of 260-350 nm, the cuvette 129 with a $1 \mathrm{~cm}$ path length was used at a protein concentration of 130 $1.5 \mathrm{mg} / \mathrm{mL}$ (40 $\mu \mathrm{M}$ monomer).

131

Enzyme Kinetic Studies. Activity of IPMDH (wild type, 132 6-12 $\mu \mathrm{g} / \mathrm{mL}$, i.e., $0.16-0.32 \mu \mathrm{M}$ monomer; or various 133 mutants, ranging from 0.1 to $10 \mathrm{mg} / \mathrm{mL}$, i.e., $2.6-260 \mu \mathrm{M}) 134$ was assayed in the presence of $0.5 \mathrm{mM} \mathrm{IPM}, 0.5 \mathrm{mM} \mathrm{MnCl}, 135$ and $4 \mathrm{mM}$ NAD in $25 \mathrm{mM}$ MOPS-KOH buffer ( $\mathrm{pH} \mathrm{7.6).} 136$ When required (e.g., in the cases of K185A, D241A, and 137 D245A mutants), the activities were assayed in the presence of 138 excess (up to $500 \mathrm{mM}$ ) $\mathrm{KCl}$. The formation of $\mathrm{NADH}$ was 139 recorded spectrophotometrically at $340 \mathrm{~nm}$ and $20{ }^{\circ} \mathrm{C}$ using a 140 Jasco (Tokyo, Japan) V-550 spectrophotometer equipped with 141 a Grant Y6 thermostat. The substrate $K_{\mathrm{m}}$ values were 142 determined at varying concentrations of each substrate, while 143 keeping constant and closely saturating concentrations of the 144 other substrates. The kinetic data were fitted using the 145 Michaelis-Menten equation.

146

Dependence of Enzyme Activity on pH. Enzyme 147 activities were tested at various $\mathrm{pHs}$ using the following 148 buffers: $25 \mathrm{mM}$ MES-NaOH ( $\mathrm{pH}$ range of 6.0-6.6), $25 \mathrm{mM} 149$ MOPS-NaOH (pH range of 6.0-8.0), $10 \mathrm{mM}$ HEPES-NaOH 150 ( $\mathrm{pH}$ range of $7.0-8.0), 50 \mathrm{mM}$ Tris- $\mathrm{HCl}(\mathrm{pH}$ range of $7.5-151$ 9.0 ), and $50 \mathrm{mM}$ diethanolamine- $\mathrm{HCl}$ ( $\mathrm{pH}$ range of $8.7-10.0)$. 152 The overlapping $\mathrm{pH}$ ranges of the particular buffers assured 153 elimination of any influence of the specific buffer components 154 on enzyme activity. To test whether substrates are applied at 155 closely saturating concentrations, the measurements at each pH 156 were repeated at various high concentrations of each substrate. 157 The experimental activity values as a function of $\mathrm{pH}$ were 158 determined and fitted according to the Henderson-Hasselbach 159 equation of a simple deprotonation dissociation curve:

$$
\nu_{\text {measured }}=\frac{\nu_{\text {extrapolated }}}{1+10^{-(\mathrm{pH}-\mathrm{pK})}}
$$

where $v_{\text {measured }}$ is the the activity value measured at a given $\mathrm{pH}, 162$ $v_{\text {extrapolated }}$ is the maximal activity value extrapolated to the high 163 $\mathrm{pH}$ range, and $\mathrm{pK}$ is the characteristic of a catalytic dissociating 164 basic or acidic side chain involved in the catalytic reaction. 165

FRET Measurements. The Förster resonance energy 166 transfer (FRET) between $\operatorname{Trp}(\mathrm{s})$ of IPMDH and the bound 167 $\mathrm{NADH}$ was recorded at $20^{\circ} \mathrm{C}$ in the presence of $\mathrm{Mg}^{2+}-\mathrm{IPM}$ as 168 reported by Dean and Dvorak ${ }^{10}$ using a SPEX Fluoromax-3 169 spectrofluorimeter equipped with a Peltier (Edison, NJ) 170 thermostat. The usual mixture contained $24 \mu \mathrm{g} / \mathrm{mL}(0.64171$ $\mu \mathrm{M}$ monomer) IPMDH (wild type or mutant) $12 \mu \mathrm{M} \mathrm{NADH}, 172$ $3 \mathrm{mM} \mathrm{MgCl}_{2}, 1 \mathrm{mM}$ IPM , and excess ( $\left.\leq 500 \mathrm{mM}\right) \mathrm{KCl}$. The 173 protein was excited at $295 \mathrm{~nm}$, and the emission was recorded 174 between 300 and $550 \mathrm{~nm}$ in a cuvette with a path length of 10175 
$176 \mathrm{~mm}$. Slits of 2 and $4 \mathrm{~nm}$ were applied for excitation and 177 emission, respectively.

178 SAXS Measurements and Data Processing. Synchro179 tron radiation solution X-ray scattering data were collected on 180 beamline P12 at the Hamburg EMBL Outstation (on the 181 PETRA III storage ring, at DESY). Solutions of complexes of 182 the wild type and the various mutants of Tt-IPMDH with $183 \mathrm{Mn}^{2+}$-IPM and NADH (nonfunctioning complex) in $25 \mathrm{mM}$ 184 MOPS-NaOH buffer ( $\mathrm{pH}$ 7.6) (cf. Table 2) were measured at 185 protein concentrations in the range of $5.0-10 \mathrm{mg} / \mathrm{mL}$ using a 186 pixel 2M PILATUS detector (DECTRIS) at a sample-detector 187 distance of $3.1 \mathrm{~m}$ and a wavelength $\lambda$ of $1.25 \AA$, covering the 188 momentum transfer $(s)$ range of $0.01-0.45 \AA^{-1}[s=4 \pi \sin (\theta)$ / $189 \lambda$, where $2 \theta$ is the scattering angle]. The concentrations of $190 \mathrm{Mn}^{2+}$, IPM, and NADH in the protein samples were $1,0.5$, and $1915 \mathrm{mM}$, respectively. To check for radiation damage, results of 19220 exposures of $50 \mathrm{~ms}$ each were compared; no radiation 193 damage effects were observed. The data, after normalization to 194 the intensity of the incident beam, were averaged, and the 195 scattering of the buffer was subtracted. All data manipulations 196 were performed using the program package PRIMUS. ${ }^{33}$

197 Structural parameters, forward scattering $I(0)$, and the radius 198 of gyration $R_{\mathrm{g}}$ were evaluated using the Guinier approxima199 tion $^{34}$ and the program GNOM. ${ }^{35}$ The radii of gyration and the 200 scattering patterns from the crystallographic models of wild201 type apo Tt-IPMDH [Protein Data Bank (PDB) entry 2Y3Z] 202 and its $\mathrm{Tt}$-IPMDH- $\mathrm{Mn}^{2+}-\mathrm{IPM}-\mathrm{NADH}$ substrate complex 203 (PDB entry 4F7I) were computed using the program 204 CRYSOL. $^{36}$ The program OLIGOMER ${ }^{33}$ was used to calculate 205 the ratio of open- and closed-form species present in $\mathrm{Tt}$ 206 IPMDH solutions as described previously. ${ }^{37}$

207 Molecular Graphical Analysis of the X-ray Structure. 208 The X-ray coordinates of the completely closed structure of the $209 \mathrm{Tt}$-IPMDH$-\mathrm{Mn}^{2+}-\mathrm{IPM}-\mathrm{NADH}$ quaternary complex (PDB 210 entry 4F7I) were used for molecular graphical analysis with the 211 aid of Insight II 95.0 (Biosym/MSI, San Diego, CA). The upper 212 distance limit for hydrogen bonds was taken to be $3.5 \AA$, while 213 for hydrophobic and ionic interactions, it was considered to be $2144.5 \AA$ A.

215 MD Simulations. To assess the most likely protonation 216 state of the side chains of the D217, D241, and K185 residues 217 and of the hydroxyl group of IPM, four different systems were 218 studied and are illustrated in Figure 4A. Five-nanosecond 219 stochastic boundary MD simulations were conducted on each 220 system using the CHARMM software package ${ }^{38}$ according to 221 the previously published protocol, ${ }^{13}$ which is also described in 222 detail in the Supporting Information.

223 QM/MM Calculations. To test the contribution of the 224 experimentally mutated side chains in enhancing the catalytic 225 activity of the enzyme, the QM/MM energy profiles obtained 226 earlier for the wild-type enzyme were reinvestigated using 227 point-charge deletion analysis (differential transition state 228 analysis). ${ }^{39-45}$ This methodology allowed us to test the 229 electrostatic effect of the mutated side chains on the reaction 230 mechanism, which is considered as the basis of the catalytic

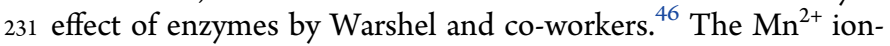
232 bound structures of the wild-type enzyme were taken from our 233 previous study. ${ }^{13}$ In that study, we showed that the proton and 234 hydride transfer reactions occur sequentially and determined 235 the reaction barrier of these processes. The effect of the 236 electrostatic environment on the reaction energetics was 237 sampled by investigating the reaction mechanism starting 238 from three structures taken from the trajectory of a $5 \mathrm{~ns}$ molecular dynamics simulation. From the three starting 239 structures, three parallel energy profiles (profiles 1, 2, and 3) 240 were determined. In this work, all three parallel profiles were 241 reinvestigated in the case of the WT and mutated enzymes. The 242 representative structures of the reactant (R), intermediate 243 ( $\left.\mathrm{I}_{\text {hydride }}\right)$, transition state (TS1 and TS2), and product $\left(\mathrm{P}_{\text {hydride }}\right), 244$ which is the product of the hydride transfer step and at the 245 same time is the most important intermediate (I) of the overall 246 catalytic cycle, of the Y139A, D217A, D241A, D245A, and 247 N102A point mutants were generated by introducing the 248 corresponding mutation in silico by annihilating the charges of 249 the corresponding residues leaving an alanine residue in the 250 systems. This ensured that the observed effects are due to the 251 electrostatic effect of the investigated mutations and do not 252 arise from sampling problems. This procedure yielded three- 253 three structures for each state $\left[\mathrm{R}, \mathrm{TS}, \mathrm{I}_{\text {hydride, }} \mathrm{TS} 2\right.$, and 254 $\left.\mathrm{I}\left(\mathrm{P}_{\text {hydride }}\right)\right]$ and for each mutant (altogether $3 \times 5 \times 5=75255$ structures). Then we conducted $\mathrm{QM} / \mathrm{MM}$ energy calculations 256 at the B3LYP/6-31G*/MM level of theory using the 257 QoMMMa program ${ }^{47}$ that couples the input and output files 258 generated by the Gaussian $09^{48}$ and TINKER $^{49}$ program 259 packages. The quantum mechanically described region included 260 the IPM molecule, the nicotinamide ring of $\mathrm{NAD}^{+}$with the 261 ribose moiety directly attached to it, $\mathrm{K} 185^{\prime}$ (the prime denotes 262 the side chain from the other subunit), and a water molecule 263 (w2013) located close to both IPM and K185' (this 264 corresponds to the QM1 region in our previous work ${ }^{13}$ ). In 265 the work presented here, we decided to use this QM region that 266 is smaller than the one used in our previous work (QM2) 267 because several mutated amino acids belong to the first 268 coordination sphere of $\mathrm{Mn}^{2+}$ and as a consequence the 269 manganese ion would lack important ligands, and if it was 270 described quantum mechanically, which would most likely lead 271 to erroneous results. However, this problem does not arise if 272 $\mathrm{Mn}^{2+}$ is described by molecular mechanics, more specifically by 273 its charge and Lennard-Jones parameters that usually provide a 274 good description of the nonbonding interaction.

275

Once the relative energy of each state along the three parallel 276 profiles calculated for each mutated enzyme was determined, 277 we calculated how much the relative energy of each state is 278 modified by the mutations using eq 2 :

279

$$
\Delta \Delta E_{\text {state,profile }}^{\text {mutant }}=\Delta E_{\text {state,profile }}^{\text {mutant }}-\Delta E_{\text {state,profile }}^{\mathrm{WT}}
$$

over all states $\left(\mathrm{R}, \mathrm{TS} 1, \mathrm{I}_{\text {hydride, }}\right.$ TS2, and $\left.\mathrm{P}_{\text {hydride }}\right)$, all profiles $(1-281$ 3 ), and all mutants. After this, we calculated the average value 282 $\left(\overline{\Delta \Delta E}_{\text {state }}^{\text {mutant }}\right)$ and the standard deviation (SD) of these 283 increments over the three profiles and added them to the 284 relative energies calculated for the corresponding WT 285 structures for profile $2\left(\Delta E_{\text {state, } 2}^{\mathrm{WT}}\right)$ (see eq 3) to obtain the 286 relative energy of the mutant enzyme $\left(\Delta E_{\text {state }}^{\text {mutant }}\right)$ in a given 287 state:

$$
\Delta E_{\text {state }}^{\text {mutant }}=\Delta E_{\text {state }, 2}^{\mathrm{WT}}+\overline{\Delta \Delta E}_{\text {state, }}^{\text {mutant }}
$$

From the obtained activation energy differences between the 290 wild-type and point-mutated enzymes, the capability of the 291 studied side chains to stabilize and/or destabilize the transition 292 state via electrostatic interactions can be estimated. If the 293 annihilation of the charges of a given residue leads to higher 294 activation energies, it implies that the given side chain is 295 electrostatically responsible for stabilization of the transition 296 state and is important for catalysis. On the other hand, if no 297 significant increase in the activation energy is observed, it 298 
A

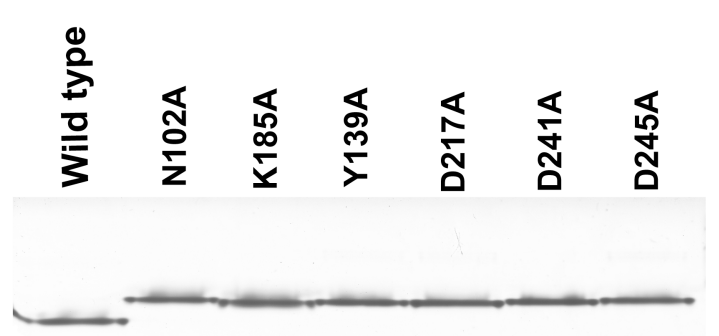

B

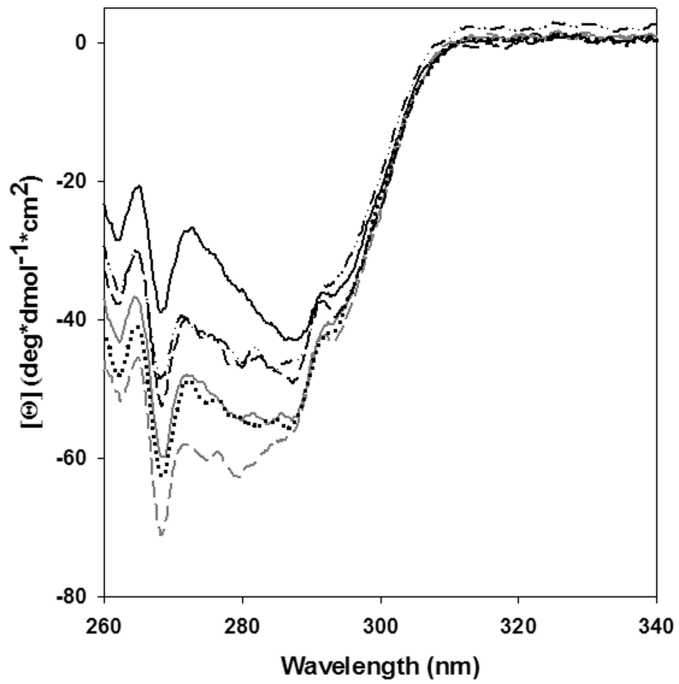

Figure 1. (A) Native gel electrophoresis pattern and (B) near-UV CD spectra of the investigated active site mutants of Tt-IPMDH. The experimental details are given in Materials and Methods. In panel B, the near-UV CD spectra of the wild-type enzyme (-) as well as mutants D241A (---), D217A ( ..), D245A (-•-), K185A (gray line), and Y139A (gray dashed line) are illustrated.

Table 1. Kinetic Parameters of the Various Single-Side Chain Mutants of Tt-IPMDH ${ }^{a}$

\begin{tabular}{|c|c|c|c|c|c|c|}
\hline$T t$-IPMDH & $k_{\text {cat }}\left(\min ^{-1}\right)$ & $K_{\mathrm{m}}^{\mathrm{IPM}}(\mu \mathrm{M})$ & $K_{\mathrm{m}}^{\mathrm{NAD}^{+}}(\mu \mathrm{M})$ & $K_{\mathrm{m}}^{\mathrm{Mn}^{2+}}(\mu \mathrm{M})$ & $\mathrm{pK}$ of $\mathrm{pH}$ dependence & $\Delta \Delta E^{\ddagger}(\mathrm{kcal} / \mathrm{mol})$ \\
\hline wild type ${ }^{b}$ & $240 \pm 30(100 \%)$ & $16 \pm 3$ & $290 \pm 50$ & $10 \pm 4$ & $7.4 \pm 0.1$ & 0.0 \\
\hline $\mathrm{K} 185 \mathrm{~A}$ & $0.15 \pm 0.05(0.06 \%)$ & $145 \pm 30$ & $64 \pm 7$ & $8 \pm 2$ & $8.4 \pm 0.2$ & 4.3 \\
\hline Y139A & $6.5 \pm 2.0(2.7 \%)$ & $40 \pm 9$ & $60 \pm 12$ & $28 \pm 5$ & - & 2.1 \\
\hline D217A & $2.7 \pm 0.5(1.1 \%)$ & $13 \pm 3$ & $305 \pm 50$ & $49 \pm 7$ & $7.9 \pm 0.1$ & 2.6 \\
\hline D241A & $0.15 \pm 0.05(0.06 \%)$ & $255 \pm 40$ & $320 \pm 50$ & $48 \pm 8$ & $8.5 \pm 0.2$ & 4.3 \\
\hline D245A & $26 \pm 8(10.9 \%)$ & $20 \pm 5$ & $375 \pm 60$ & $23 \pm 5$ & $7.9 \pm 0.1$ & 1.3 \\
\hline N102A & $33 \pm 8(13.9 \%)$ & $14 \pm 5$ & $260 \pm 80$ & $32 \pm 5$ & $\mathrm{nd}^{d}$ & 1.1 \\
\hline $\mathrm{E} 270 \mathrm{~A}^{c}$ & $1.96 \pm 0.3(0.8 \%)$ & $32 \pm 5$ & $660 \pm 55$ & $25 \pm 6$ & $\mathrm{nd}^{d}$ & 2.8 \\
\hline
\end{tabular}

${ }^{a}$ Enzyme activities of the wild type and the various mutant forms of IPMDH were measured as described in Materials and Methods. The pK values were determined from the $\mathrm{pH}$ dependences of the activities by fitting the values to eq 1 . The increase in activation energy compared to the wild-type enzyme $\left[\Delta \Delta E^{\ddagger}=-R T \ln k_{\text {cat }}(\right.$ mutant $) / k_{\text {cat }}$ (wild)] was estimated from the $k_{\text {cat }}$ values using the Arrhenius equation and is given in kilocalories per mole. ${ }^{b}$ Determined previously. ${ }^{37,57,62}{ }^{c}$ Determined previously. ${ }^{57}{ }^{d}$ Not determined.

299 implies that the given residue does not contribute electrostati300 cally to transition state stabilization of the hydride transfer; 301 however, this does not mean that it cannot be important for 302 catalysis (e.g., through mediation of a protein conformational 303 shift or contributing to the decarboxylation step in the present 304 enzyme), just that our method cannot capture this effect.

\section{RESULTS AND DISCUSSION}

306 Physicochemical Properties of the Active Site 307 Mutants of Tt-IPMDH. SDS-PAGE of the Ala mutants of 308 selected active site residues of $T t$-IPMDH illustrated their 309 satisfactory purity. Their native gel electrophoretic pattern 310 further indicates that all the mutants retain the dimeric nature, 311 albeit with a somewhat increased hydrodynamic radius that 312 results in a small but significant decrease in their electro313 phoretic mobility compared to that of the wild-type enzyme 314 (Figure 1A). We have checked that the contribution of the 315 active site Lys and Asp residues to the global surface charge of 316 the protein molecule is negligible under our experimental 317 conditions. Thus, we cannot expect easily detectable changes in 318 the electrophoretic running pattern upon mutation of the active 319 site Lys and Asp side chains into Ala itself. Therefore, the 320 observed decrease in the electrophoretic mobilities of all the 321 mutants (relative to the wild-type enzyme) might be due to a small perturbation of the tertiary and/or quaternary structure of 322 the protein molecule. Changes in the relative positions of the 323 two domains (e.g., domain opening) could equally lead to a 324 unidirectional increase in the molecular dimensions and a 325 consequent reduction of the electrophoretic mobility. Our 326 SAXS measurements (cf. below), indeed, supported this 327 proposal. At the same time, the near-UV CD spectra of the 328 mutants exhibit slight but significant differences from that of 329 the wild-type enzyme, consistent with small local changes in the 330 tertiary structure upon mutation (Figure 1B). On the other 331 hand, the far-UV CD spectra of the mutants do not differ from 332 that of the wild-type enzyme, indicating the identities of their 333 secondary structures (not shown). In agreement, all the 334 mutants exhibited cooperative heat transitions by DSC 335 calorimetry, with transition temperatures similar to that of 336 the wild-type enzyme (not illustrated).

Effects of Mutation of the Active Site Residues on the 338 Kinetic Properties of Tt-IPMDH. Table 1 summarizes the $339 \mathrm{t} 1$ kinetic constants of the investigated single side-chain mutants 340 of Tt-IPMDH as compared to the values characteristic of the 341 wild-type enzyme. The largest decreases in the $k_{\text {cat }}$ value have 342 been observed in the cases of K185A and D241A mutants, both 343 of them exhibiting only $0.06 \%$ of the wild-type enzyme activity. 344 Activities of the other mutants decrease in the following order: 345 
346 N102A (13\%) > D245A (10.5\%) > Y139A (2.9\%) > D217A $347(1.1 \%)$. The $K_{\mathrm{m}}$ values of the substrates do not show similarly 348 large changes; only the $K_{\mathrm{m}}{ }^{\mathrm{IPM}}$ values of K185A and D241A 349 mutants with the lowest catalytic activities increase appreciably, 350 indicating weakening of the catalytic interactions with IPM.

351 However, the $K_{\mathrm{m}}{ }^{\mathrm{NAD}^{+}}$values do not exhibit appreciable changes 352 for either mutant, because all of the mutations are located in the 353 IPM binding site. The only exception is the Y139A mutant that 354 unexpectedly exhibits a significant decrease in its $K_{\mathrm{m}}{ }^{\mathrm{NAD}^{+}}$, which 355 is similar to the observations by Miyazaki and Oshima that were 356 interpreted as an effect of some undefined local conformational 357 changes in the $\mathrm{NAD}^{+}$binding site of this mutant. ${ }^{52}$ It may be 358 notable that previously we have detected a significant increase 359 in $K_{\mathrm{m}} \mathrm{NAD}^{+}$in the case of mutation of E270, the glutamate side 360 chain of which directly interacts with $\mathrm{NAD}^{+}$. For comparison, 361 the kinetic data of E270A are also listed in Table 1.

362 Figure 2 illustrates the positions and the interactions of the 363 presently mutated side chains in the IPM binding site. Among

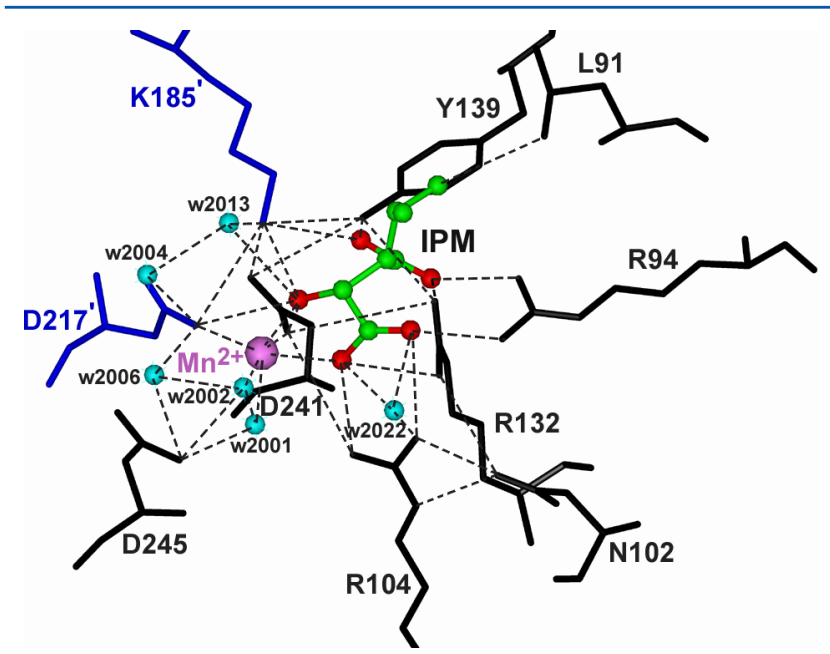

Figure 2. Structural details of binding of $\mathrm{Mn}^{2+}-\mathrm{IPM}$ to $T t$-IPMDH in the completely closed $\mathrm{Mn}^{2+}-\mathrm{IPM}-\mathrm{NADH}-\mathrm{IPMDH}$ quaternary complex. Using the atomic coordinates of PDB entry 4F7I of the wild-type quaternary complex of $\mathrm{Tt}$-IPMDH, ${ }^{13}$ the substrate binding site is depicted as follows. The active site side chains are illustrated by black and blue stick models indicating their origins of different subunits. The substrate IPM is shown with ball and stick models, colored according to atom. $\mathrm{Mn}^{2+}$ and the water molecules bound in the active site are represented by purple and blue spheres, respectively. The dashed lines represent the atomic interactions (H-bonds and/or electrostatic interactions, defined in Materials and Methods; cf. Molecular Graphical Analysis of the X-ray Structure).

364 them, K185', D241, and D217' (the prime denotes the 365 positioning of the side chain in the other subunit of the dimer) 366 contact the reacting $\mathrm{OH}$ group of the substrate IPM and Y139 367 contacts the C3-carboxylate of IPM. As expected, the directly 368 contacting side chains of $\mathrm{K}^{185^{\prime}}$, D241, D217', and Y139 exhibit 369 the greatest responsibility for enzyme activity. The side chains 370 of N102 and D245 have smaller contributions to the enzyme 371 activity and no direct contacts with the substrate.

$372 \mathrm{pH}$ Dependence of the Enzyme Activities of the 373 Active Site Mutants of Tt-IPMDH. Similar to other oxidative 374 decarboxylases, ${ }^{1}$ IPMDH functions via an acid-base catalysis; 375 the $\mathrm{pH}$ dependence of the enzyme activity is an informative 376 characteristic of this aspect. Previously, we have characterized 377 this property of wild-type Tt-IPMDH and attributed it to the dissociation of an ionizable group with a $\mathrm{pK}$ of $7.4 .^{53}$ Here, the 378 same analysis has been conducted with the investigated mutants 379 (Figure 3 ), and the derived $\mathrm{pK}$ values are summarized in Table $380 \mathrm{f} 3$

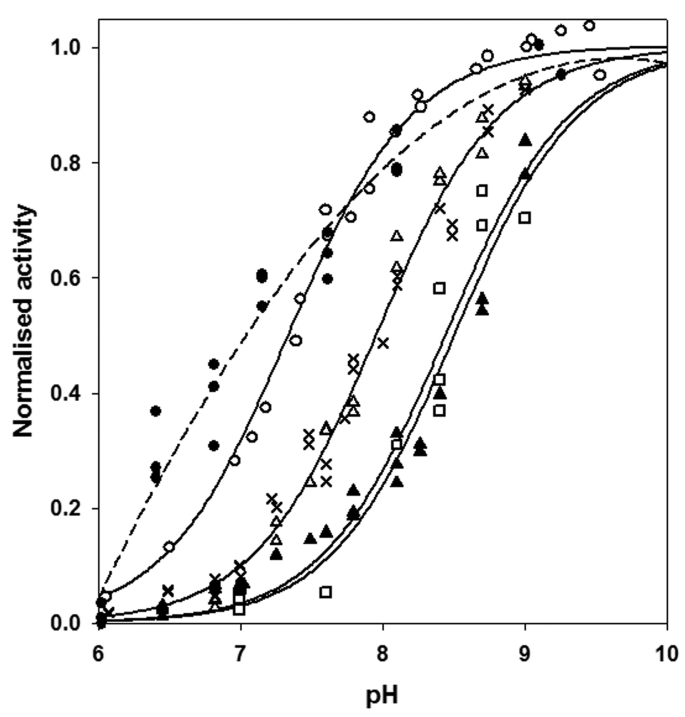

Figure 3. $\mathrm{pH}$ dependence of enzyme activities of various active site mutants of $T t$-IPMDH in comparison to that of the wild-type enzyme. Enzyme activities of wild-type IPMDH $(\mathrm{O})$ as well as mutants D241A $(\mathbf{\Delta})$, D217A $(\times)$, D245A $(\triangle)$, K185A $(\square)$, and Y139A (•) are plotted as a function of $\mathrm{pH}$ after normalization of the data to the same maximal activities at high $\mathrm{pH}$ values. The data were fitted to eq $1(-)$, and the obtained $\mathrm{pK}$ values are summarized in Table 1 . The dashed line illustrates the $\mathrm{pH}$ dependence of activity of Y139A that does not follow a simple $\mathrm{pK}$ curve.

1. Only the $\mathrm{pH}$ dependence of the activity of Y139A did not 381 follow a simple ionization curve. Because the CD spectra of the 382 enzymes [recorded in the $\mathrm{pH}$ range of 6-9 (not shown)] do 383 not indicate any significant structural changes, the observed $\mathrm{pH} 384$ dependences of the activities can most probably be due to the 385 ionization of at least one specific active site residue. 386

Remarkably, the two mutants (K185A and D241A) 387 exhibiting the lowest catalytic activities are characterized by 388 the largest shift of their $\mathrm{pK}$ values toward the more basic region. 389 Moreover, the $\mathrm{pH}$ dependences of their activities are almost 390 coincident. The first finding clearly supports our previous 391 conclusion from a combined crystallographic and QM/MM 392 modeling study that the $\mathcal{E}$-amino group of $\mathrm{K} 185^{\prime}$ acts as a 393 general base in the catalysis aiding the deprotonation reaction, 394 prior to the transfer of the hydride from the substrate IPM to 395 the nicotinamide ring of $\mathrm{NAD}^{+13}$ (Scheme 1). The closely $396 \mathrm{~s} 1$ similar $\mathrm{pH}$ dependences of the activities of K185A and D241A 397 may indicate that the carboxylate of D241 acts cooperatively 398 with $\mathrm{K}_{185^{\prime}}$ in the abstraction of a proton from IPM, as also 399 supported by the present QM/MM modeling study (cf. below). 400 This proton relay system may be additionally aided by several 401 $\mathrm{H}$-bonding water molecules (cf. Figure 2, including the catalytic 402 water, w2013 ${ }^{13}$ ), located in the active site. Although the 403 activity $-\mathrm{pH}$ profiles of the other two Asp mutants (D217A and 404 D245A) are somewhat different, they both exhibit an 405 intermediate $\mathrm{pK}$ value of 7.9. Still, the contributions of these 406 two active site Asp residues and the interacting water molecules 407 (Figure 2) to the proton relay system cannot be excluded. 408

No similar conclusion can be drawn from the unusual $\mathrm{pH} 409$ dependence of the activity of the Y139A mutant. The 410 equivalent Y160 of E. coli ICDH was assumed to be a general 411 
Scheme 1. Complete Reaction Mechanism of the Catalytic Cycle by IPMDH ${ }^{a}$
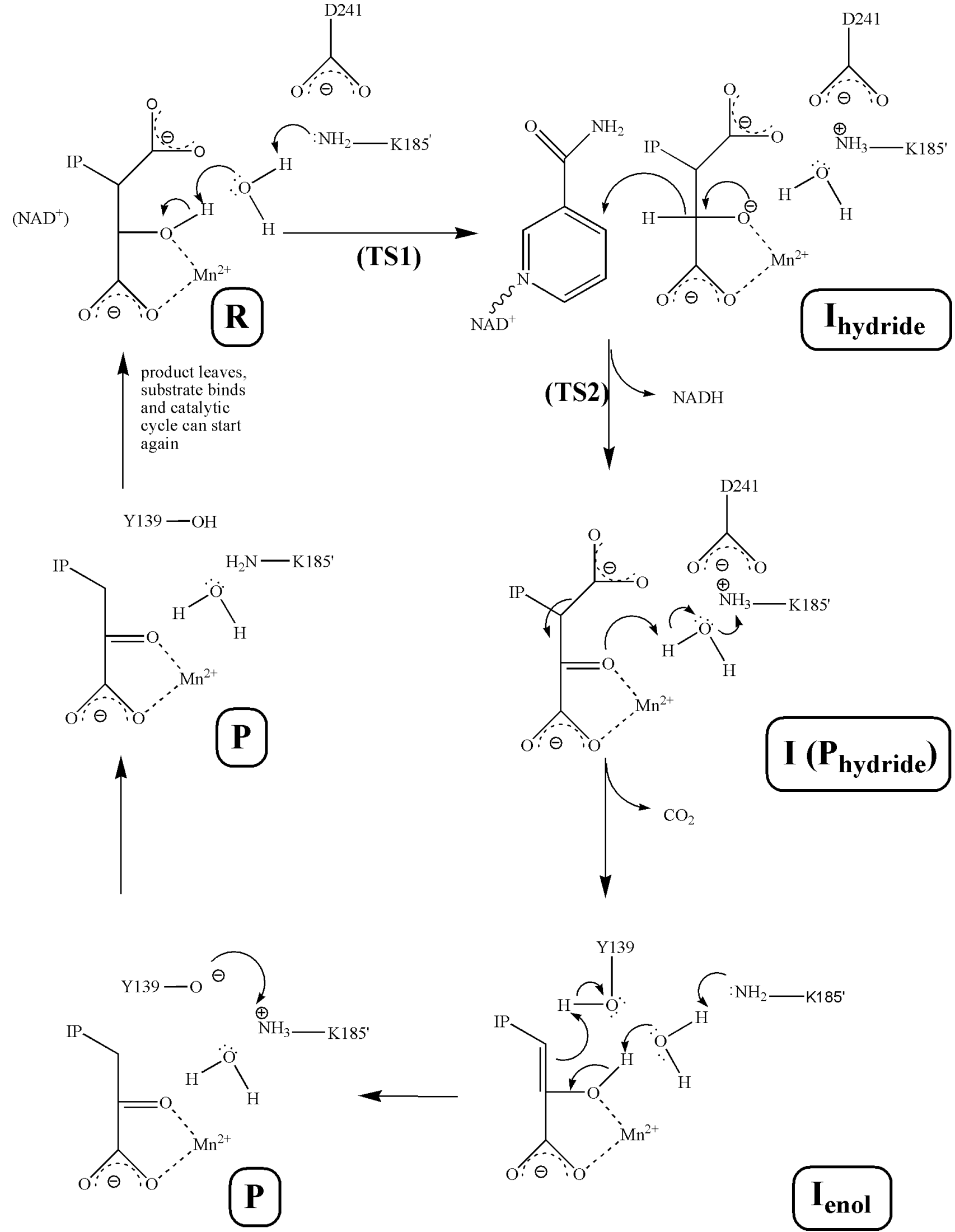

${ }^{a}$ IP represents the isopropyl group of IPM. R, I, and P represent the reactant, intermediate, and product states, respectively. It is notable that the product of the hydride transfer $\left(\mathrm{P}_{\text {hydride }}\right)$ is identical to the intermediate $(\mathrm{I})$ state of the overall reaction. TS1 and TS2 indicate the transition states of the proton and hydride transfers, respectively. 


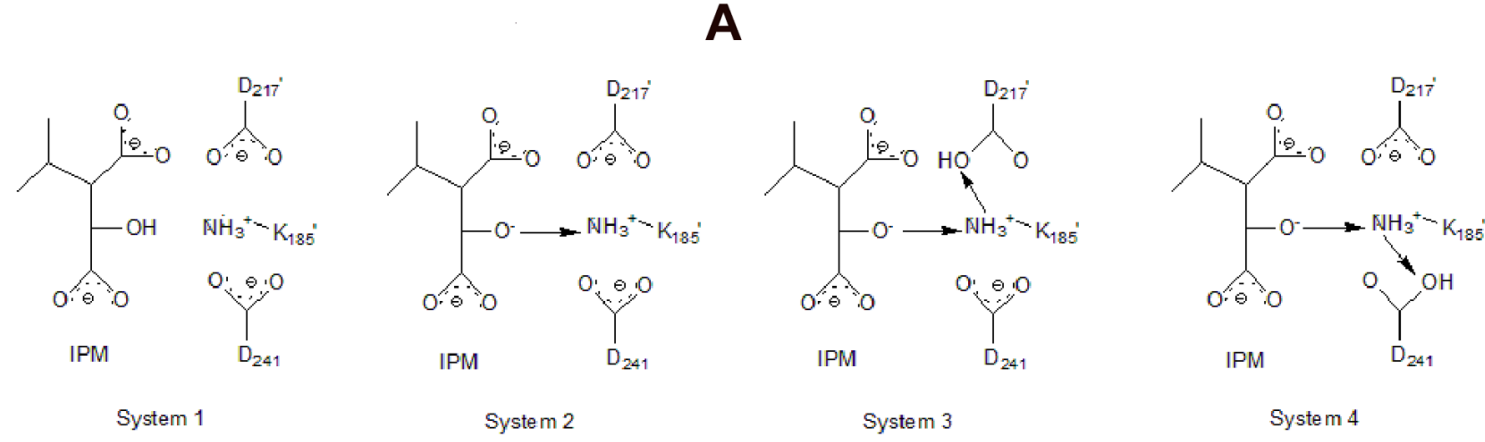

B
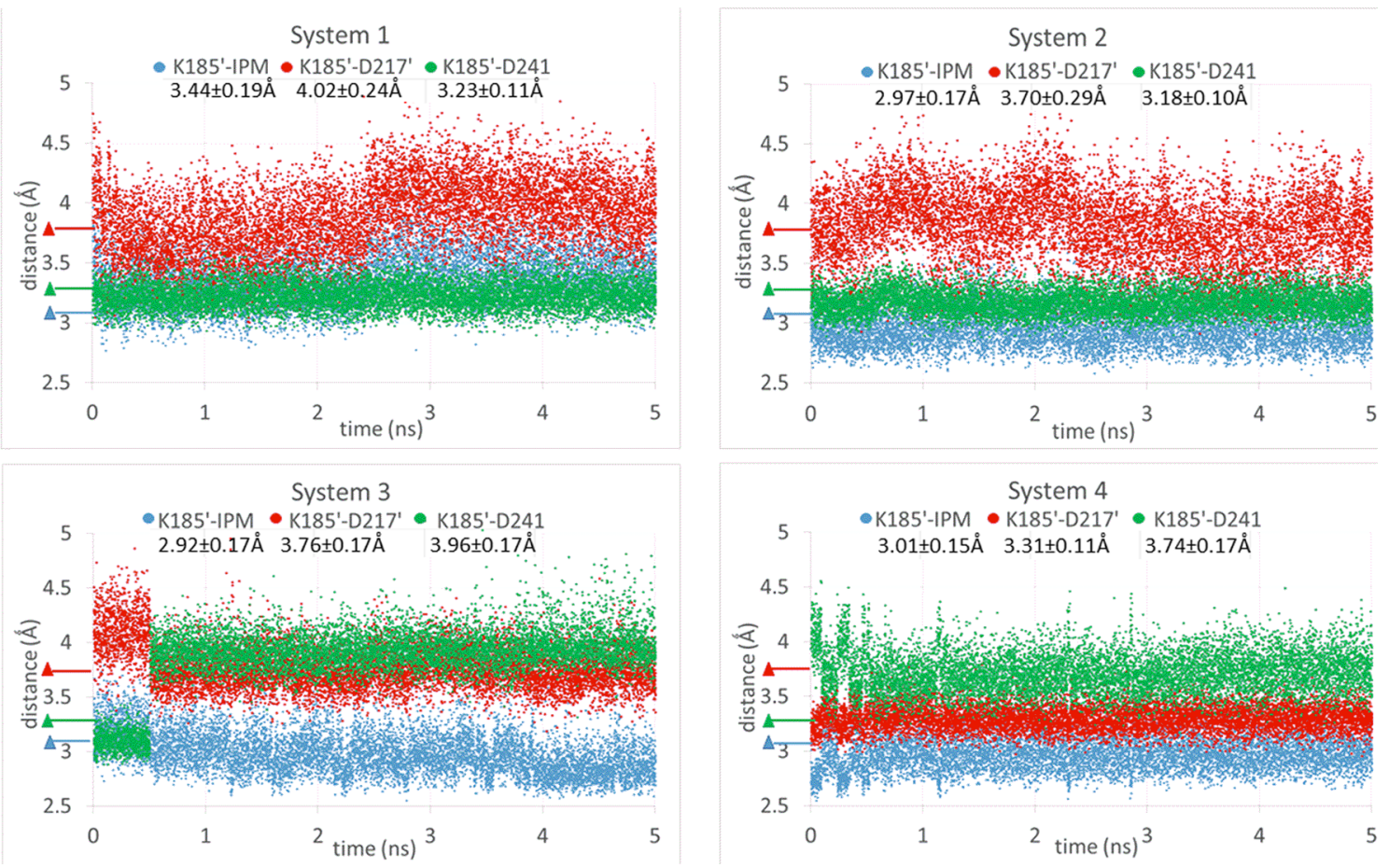

Figure 4. (A) Protonation states of IPM, K185, D217, and D241 in various systems studied by MD simulations and (B) changes in interatomic distances along the trajectory of the $\mathrm{MD}$ simulations in differently protonated systems. In panel $\mathrm{A}$, the arrows indicate the directions of proton transfers that have occurred in the investigated systems. In panel $\mathrm{B}$, in each case, the distances were measured from the $\varepsilon$-amino $\mathrm{N}$ atom of $\mathrm{K} 185^{\prime}$ to the hydroxyl O atom of IPM (blue) or to the $\gamma$-carbon atom of D217' (red) or D241 (green). In each diagram, the average distances measured during the last $2 \mathrm{~ns}$ of the simulations are numerically given together with their standard deviations. Along the $y$-axis colored triangles indicate the distance found in the corresponding X-ray structure (PDB entry 4F7I). Reference values are distances between $\varepsilon$-amino N atom of K185' and ( 1 ) the hydroxyl O atom of IPM (3.05-3.07 $\AA),(2)$ the carboxylic carbon atom of D217' (3.60-3.73 $\AA$ ), and (3) the carboxylic carbon atom of D241 (3.23-3.38 ̊̊).

412 acid in protonation of the enolate intermediate after the 413 decarboxylation step (cf. Scheme 1); this may also hold for $414 \mathrm{IPMDH}^{1}$

415 Determination of the Protonation State of K185 in 416 the Resting State of the Enzyme. On the basis of the 417 enzyme kinetic data, K185 is one of the most important 418 residues involved in the catalytic cycle of IPMDH. In our 419 previous work, ${ }^{13}$ we suggested that it was unprotonated in the 420 resting state of the enzyme. As K185 is a basic residue that is 421 considered to be protonated at ambient $\mathrm{pH}$ values, we have 422 sought to obtain further evidence to support our unusual 423 hypothesis.
One important consideration lies in comparing the number 424 of acidic (D217', D241, and D245) and basic (R94, R104, and 425 R132 apart from $\mathrm{K}^{\left.185^{\prime}\right)}$ residues in the active site. 426 Furthermore, there is a divalent cation $\left(\mathrm{Mn}^{2+} / \mathrm{Mg}^{2+}\right)$ in the 427 active site, which brings the overall charge of the active site to 428 +2 even if $\mathrm{K}^{185^{\prime}}$ is neutral. This significant positive charge in 429 the active site is needed for efficient binding of the substrate 430 IPM but does not seem to favor the presence of an extra 431 positive charge on K185'. However, the situation dramatically 432 changes when the IPM ligand (a dicarboxylic acid) binds the 433 active site: its two carboxylate groups balance the original 434 positive charge and generate better conditions for the 435 protonation of $\mathrm{K}^{185^{\prime}}$. This qualitative picture is well-supported 436 
437 by $\mathrm{p} K_{\mathrm{a}}$ calculations by the PropKa program. The $\mathrm{p} K_{\mathrm{a}}$ value of $438 \mathrm{~K}^{185^{\prime}}$ is predicted to be 5.9 in the absence and 8.1 in the 439 presence of IPM in the active site, which suggests that $\mathrm{K} 185^{\prime}$ 440 becomes protonated upon ligand binding.

441 Our hypothesis regarding the protonation state of $\mathrm{K} 185^{\prime}$ and 442 the mechanism for abstraction of a proton from the substrate is 443 in contrast to studies of other enzymes. In the case of malate 444 dehydrogenase, ${ }^{8}$ 6-phosphogluconate dehydrogenase, and 445 various isocitrate-dehydrogenase enzymes, it has been proposed 446 that the nearby lysine residue has to be first deprotonated by an 447 aspartic acid residue to be able to act as a general base to 448 remove the 2-hydroxyl proton of the substrate.

449 To decide which scenario is more likely to be valid in 450 IPMDH, we have conducted molecular dynamics simulations 451 on systems in various protonation states as shown in Figure 4A. 452 Previously, ${ }^{13}$ we have argued, on the basis of QM/MM 453 calculations, that the proton transfer process from the hydroxyl 454 group of IPM to $\mathrm{K}^{185^{\prime}}$ is very facile and is likely to occur 455 immediately upon IPM binding even if the other ligand, $\mathrm{NAD}^{+}$, 456 is not present. If this is the case, this would imply that in the 457 crystal structure of the Tt-IPMDH- $\mathrm{Mn}^{2+}-\mathrm{IPM}-\mathrm{NADH}$ 458 quaternary complex a deprotonated hydroxyl group of IPM 459 and a protonated $\mathrm{K} 185^{\prime}$ side chain should be present. As this 460 structure should be distinguishable from other differently 461 protonated systems, we conducted MD simulations to 462 investigate which protonation state is the most consistent 463 with the X-ray structure. System 1 corresponds to the reactant 464 state structure proposed for analogous enzymes: the amino 465 group of $\mathrm{K} 185^{\prime}$ is protonated, and the hydroxyl group of IPM is 466 neutral. System 2 matches our proposal: the originally neutral 467 amino group of $\mathrm{K}^{185^{\prime}}$ acted as a base and abstracted the proton 468 from the hydroxyl group of IPM. Systems 3 and 4 are 469 consistent with the proposal that $\mathrm{K} 185^{\prime}$ is protonated in the 470 resting state of the enzyme and in the course of the catalyzed 471 reaction abstracted the proton from the hydroxyl group of IPM 472 and transferred one of its protons to a nearby aspartic acid 473 residue, either to D217 (system 3) or to D241 (system 4).

474 We have monitored the distances that are characteristic of 475 the interactions among the amino group of $\mathrm{K} 185^{\prime}$, the hydroxyl 476 group of IPM, and the carboxylic groups of D217' and D241. 477 The results have been summarized in Figure 4B. It is obvious 478 from the figure that the characteristic distances show very great 479 variation with the protonation state of the studied residues, and 480 only system 2 features distances consistent with the X-ray 481 structure. In the case of system 2 , all distances vary around the 482 corresponding distances in the X-ray structure. In contrast, 483 some major rearrangements are observed for the other systems. 484 For example, in the case of system 1, the distance between the 485 amino nitrogen atom of $\mathrm{K}^{185^{\prime}}$ and the hydroxyl oxygen of IPM 486 increases to $\sim 3.5 \AA$ in contrast to the experimental value of $4873.07 \AA$. Furthermore, the hydroxyl group of IPM turns away 488 from $\mathrm{K}^{185}$, and the hydroxyl hydrogen is found in a very 489 unfavorable position for the proton transfer to occur. The 490 results of the MD simulations on systems 3 and 4 are even 491 more inconsistent with the X-ray structure, where D241 is 492 located closer to $\mathrm{K} 185^{\prime}$ (the distances of the carboxylate $\mathrm{O}$ 493 atoms from the Lys NZ atom are 3.30 and $2.62 \AA$ ) than to $494 \mathrm{D} 217^{\prime}$ (the corresponding distances are 3.78 and $3.60 \AA$, 495 respectively), but this order is reversed in these two model 496 systems. Therefore, the results of the MD simulations strongly 497 support the hypothesis that (1) in the resting state of the 498 enzyme $\mathrm{K} 185^{\prime}$ is unprotonated, (2) this residue is the final base 499 responsible for the deprotonation of the hydroxyl group of
IPM, and (3) D217' or D241 are not directly involved in the 500 proton transfer process.

As other members of the $\beta$-hydroxyacid oxidative decarbox- 502 ylase family are thought to operate by a similar mechanism, we 503 were interested to know whether the finding that the 504 catalytically active lysine residue $\left(\mathrm{K}^{185^{\prime}}\right)$ is unprotonated in 505 the resting state of IPMDH may be relevant to the other 506 members of the enzyme family. Therefore, using the PropKa 507 program, we predicted the $\mathrm{p} K_{\mathrm{a}}$ value of the catalytically active 508 lysine residues in related enzymes in the presence and absence 509 of their substrates. In each case, we observed that in the 510 presence of the substrate the $\mathrm{p} K_{\mathrm{a}}$ value of the lysine residue is 511 predicted to increase by $\sim 2$ units, similar to the case for the 512 IPMDH enzyme (see the details in the Supporting 513 Information). This suggests that the unprotonated nature of 514 the catalytic lysine residue might be a general feature of the $\beta$ - 515 hydroxyacid oxidative decarboxylase family.

516

Testing the Catalytic Role of the Active Site Side 517 Chains by QM/MM Modeling. As mentioned above, IPMDH 518 catalyzes a two-step reaction: the $\mathrm{NAD}^{+}$-dependent reversible 519 oxidation of IPM followed by an irreversible decarboxylation 520 step (cf. Scheme 1). We showed earlier that the oxidation step 521 itself is a sequential event of a proton transfer step and a 522 hydride transfer step. ${ }^{13}$ Starting from the reactant state (R), a 523 proton from the hydroxyl group of IPM is transferred via a 524 catalytic water molecule to $\mathrm{K} 185^{\prime}$ involving a low-energy 525 transition state $(\mathrm{TS} 1)$ to form an intermediate $\left(\mathrm{I}_{\text {hydride }}\right)$. From 526 this state, a hydride ion is transferred from C2 of IPM to $\mathrm{NAD}^{+} 527$ via a second transition state (TS2) to form the 3-isopropyl- $\alpha$ - 528 keto-glutarate product $\left(\mathrm{P}_{\text {hydride }}\right)$ of the oxidation step, which is 529 itself the major intermediate (I) of the overall catalytic reaction 530 and can undergo irreversible decarboxylation in the active site 531 of the enzyme.

In the study presented here, we extended our calculations to 533 rationalize the experimentally observed activities of the mutated 534 enzymes. Herein, we could not study the effect of $\mathrm{K} 185^{\prime}$ on the 535 reaction mechanism, as it acts as the proton acceptor according 536 to the proposed reaction mechanism and participates in a bond 537 forming process, which can be modeled by only quantum 538 mechanical methods. For this reason, this residue has to be 539 included in the quantum mechanically described region; thus, 540 its effect cannot be studied in the same manner as those of the 541 other side chains. Most likely, the K185A mutant enzyme 542 operates in a manner slightly different from that of the wild- 543 type enzyme; the proton acceptor role of K185 could be taken 544 over by one of the aspartate residues or possibly by a hydroxide 545 ion. Some support for the latter hypothesis is provided by the 546 fact that the $\mathrm{pH}$ optimum of the K185A enzyme is higher than 547 that of the wild-type enzyme. This is evidenced by their $\mathrm{pK} 548$ profiles: for the mutant enzyme, it is shifted toward $\mathrm{pH}$ values 549 (thus to higher hydroxide ion concentrations) higher than that 550 of the wild-type enzyme.

551

Thus, using point-charge deletion analysis, we studied the 552 effect of N102A, Y139A, D217A, D241A, and D245A 553 mutations on the proton and hydride transfer steps of the 554 catalytic reaction. This approach allowed us to determine 555 whether the experimentally observed decrease in the $k_{\text {cat }}$ values 556 of the mutants is likely to originate from a direct effect or an 557 indirect effect. In the first case, the residue has a major role in 558 stabilizing the transition state, and this should be reflected in 559 the obtained activation energies. However, indirect effects (e.g., 560 when mutation perturbs the tertiary structure of the enzyme) 561 cannot be captured by point-charge deletion analysis: they 562 
563 would require extended molecular dynamics simulations to 564 identify the exact role of the residue in the tertiary structure and 565 how it reduces the catalytic activity. Nevertheless, in real 566 systems, a mixture of these effects may be the reason for the 567 decreased catalytic activity.

568 Before we discuss the obtained results, it might be useful to 569 touch upon the accuracy of our calculated data and emphasize 570 that the obtained numbers give only qualitative insight and 571 should not be taken literally. On the basis of the Arrhenius 572 equation, the activation energy differences between the wild573 type and mutant enzymes can be predicted from the 574 experimental $k_{\text {cat }}$ values (see Table 1). The data show that 575 the experimentally observed reduced reaction rates do not 576 always translate into a seriously increased energy of activation; 577 e.g., the $13.9 \%$ activity of the N102A mutant corresponds to an 578 only $1.1 \mathrm{kcal} / \mathrm{mol}$ increase in the activation energy. 579 Unfortunately, this effect, even with very precise computational 580 methods [e.g., with the $\operatorname{CCSD}(\mathrm{T})$ method, which is the gold 581 standard of computational chemistry], cannot be expected to be 582 described reliably. In light of the estimated activation energy 583 differences in the various mutants, one can realistically expect 584 the calculations to reproduce that of the D241A mutation as 585 the most severe one; the Y139A and D217A mutants should 586 have similar but less reduced activities, and the D245A and 587 N102A mutations should have an even smaller effect on the 588 catalytic reaction.

589 In Figure 5A, we have plotted the estimated relative energies 590 of the most important structures involved in the proton and

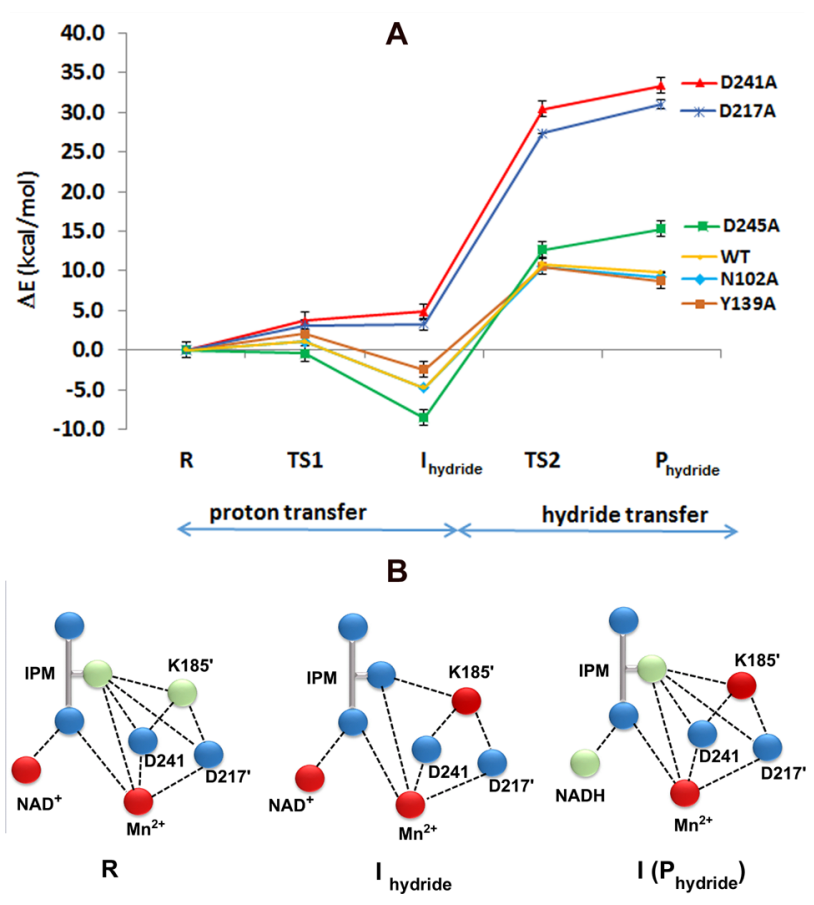

Figure 5. Results and interpretation of the QM/MM calculations. The estimated relative energies with their standard deviations $\left(\Delta E_{\text {state }}^{\text {mutant }}\right.$; cf. eq 3) of the various states (reactant R, transition states TS1 and TS2, intermediate $\mathrm{I}_{\text {hydride, }}$ and product $\mathrm{P}_{\text {hydride }}$ ) of the proton and hydride transfer reactions of the mutant variants of $T t$-IPMDH are represented in panel A. The major electrostatic interactions of the active site are illustrated in panel B in the reactant $(\mathrm{R})$ and the intermediate $\left(\mathrm{I}_{\text {hydride }}\right)$ states during the hydride transfer reaction. The differently charged groups are colored differently (green, neutral; blue, negative; and red, positive) hydride transfer step. With the exception of the D241A and 591 D217A mutants, all three profile calculations show exactly the 592 same trend and the data are characterized by a very small 593 standard deviation. However, the results with D241A and 594 D217A are found to be contradictory. Two profiles (profiles 2595 and 3 referring to our previous work with the wild-type 596 enzyme $^{13}$ ) suggested a more significant role for the D241A 597 mutation than for the D217A mutation, in accordance with the 598 experiment, but profile 1 predicted the opposite effect (cf. also 599 eq 2). Therefore, we have carefully checked the structures, and 600 we found that in the case of the structural model corresponding 601 to profile 1 the interaction between D241 and K185' is 602 significantly different from that of the other two profiles as well 603 as from that of the $\mathrm{X}$-ray structure. As mentioned in the 604 previous chapter, in the X-ray structure (as well as in the 605 structural models for profiles 2 and 3), D241 is located 606 definitely closer to $\mathrm{K}^{2} 85^{\prime}$ than $\mathrm{D} 217^{\prime}$, and both of the 607 carboxylic oxygen atoms of D241 interact with K185'. In 608 contrast in profile 1, D241 turned away from $\mathrm{K} 185^{\prime}$; only one 609 of its carboxylate oxygen atoms remained in the vicinity, leading 610 to false results. For this reason in Figure 5A for D217A and 611 D241A, the data obtained from only profiles 2 and 3 are 612 reported. In the case of both D217A and D241A, almost 613 monotonously increasing relative energy curves are predicted 614 by the calculations with an even greater effect for the D241A 615 mutation, in accordance with the experiment. However, the 616 monotonously increasing nature of the curves is apparently in 617 contrast to the expectation that in a chemically viable reaction 618 the energy of the transition state should be higher than that of 619 the product (e.g., compare TS2 with Phydride). It is important 620 to emphasize that the obtained numbers do not describe the 621 exact thermodynamical properties of the mutant enzymes; 622 instead, they shed light on the electrostatic effect of the 623 investigated amino acids in the wild-type enzyme, from which 624 one may obtain an approximate view on the functioning of the 625 mutant enzymes. This means that when D241 or D217' is 626 replaced with Ala a very important stabilizing group is removed 627 from the active site of the enzyme, which leads to very high 628 relative energies of the states involved in the hydride transfer 629 step $\left(\mathrm{I}_{\text {hydride, }}\right.$ TS2, and $\left.\mathrm{P}_{\text {hydride }}\right)$ and that this effect not only is 630 essential in the transition state but also contributes to the 631 stabilization of the Phydride state, which is the intermediate of 632 the overall catalytic cycle. This implies that these groups are 633 essential for the proper functioning of the enzyme in 634 accordance with the results of activity measurements, which 635 showed very low activities for these mutants [D217A, 1.1\%; 636 D241A, $0.06 \%$ (cf. Table 1)]. Within the accuracy of the 637 calculations, the activity difference between these two mutants 638 is reasonably well reproduced. The fact that the chemical 639 reaction occurs in these mutant enzymes suggests that most 640 likely the intermediate and transition state structures of the 641 mutant enzymes are different from those of the wild type; i.e., 642 new reaction routes might be opened to compensate for the 643 effect of the mutations. However, these possibilities were 644 outside of the scope of the modeling presented here. 645

In Figure 5B, we have pictorially demonstrated the basis for 646 the considerable catalytic effect of these two side chains in the 647 WT enzyme. In the reactant state, $\mathrm{K} 185^{\prime}$ is neutral and interacts 648 via hydrogen bonding interactions with D217' and D241. 649 These interactions increase the basicity of $\mathrm{K}^{185}$ ', which can 650 deprotonate the hydroxyl group of IPM via TS1 leading to 651 $\mathrm{I}_{\text {hydride, }}$ which in turn is stabilized by numerous favorable 652 electrostatic interactions. When D217' or especially D241, 653 
Table 2. Comparison of SAXS Experimental Data of the Tt-IPMDH-Mn ${ }^{2+}-\mathrm{IPM}-\mathrm{NADH}$ Quaternary Complex with Those Derived from the Crystallographic Models

\begin{tabular}{|c|c|c|c|c|c|c|c|}
\hline & \multicolumn{2}{|c|}{$R_{\mathrm{g}}(\AA)^{a}$} & \multicolumn{3}{|c|}{$\begin{array}{c}\text { discrepancy }^{d} \text { values } \chi \text { between the scattering from crystallographic } \\
\text { models and experimental data }\end{array}$} & \multicolumn{2}{|c|}{$\begin{array}{c}\text { volume fractions of } \\
\text { open/closed } \\
\text { structures }\end{array}$} \\
\hline & GNOM method & Guinier method & closed crystal structure & open crystal structure & open/closed mixture ${ }^{e}$ & $V_{\text {closed }}(\%)$ & $V_{\text {open }}(\%)$ \\
\hline wild type & $27.4 \pm 0.2$ & $27.4 \pm 0.3$ & 1.18 & 1.19 & 1.09 & $53 \pm 3$ & $47 \pm 3$ \\
\hline K185A mutant & $28.1 \pm 0.2$ & $28.2 \pm 0.3$ & 2.26 & 1.19 & 1.15 & $15 \pm 3$ & $85 \pm 3$ \\
\hline Y139A mutant & $27.6 \pm 0.2$ & $27.7 \pm 0.3$ & 1.53 & 1.28 & 1.22 & $46 \pm 3$ & $54 \pm 3$ \\
\hline D217A mutant & $28.4 \pm 0.2$ & $28.5 \pm 0.3$ & 2.41 & 1.22 & 1.22 & 0 & 100 \\
\hline D241A mutant & $28.4 \pm 0.2$ & $28.5 \pm 0.3$ & 2.49 & 1.26 & 1.26 & 0 & 100 \\
\hline D245A mutant & $27.9 \pm 0.2$ & $28.0 \pm 0.3$ & 2.16 & 1.41 & 1.32 & $32 \pm 3$ & $68 \pm 3$ \\
\hline N102A mutant & $28.2 \pm 0.2$ & $28.3 \pm 0.3$ & 2.32 & 1.36 & 1.16 & $22 \pm 3$ & $78 \pm 3$ \\
\hline E270A mutant ${ }^{b}$ & $28.4 \pm 0.2$ & $28.5 \pm 0.3$ & 1.71 & 1.08 & 1.07 & $5 \pm 3$ & $95 \pm 5$ \\
\hline$R_{\mathrm{g}}($ theoretical $)(\AA)^{c}$ & 26.67 & 28.45 & & & & & \\
\hline molecular mass $(\mathrm{kDa})^{c, g}$ & 74.3 & 73.8 & & & & & \\
\hline
\end{tabular}

${ }^{a}$ The $R_{\mathrm{g}}$ values were computed by two alternative methods, using the program GNOM and Guinier approximation. ${ }^{b}$ Published previously. ${ }^{57}{ }^{c}$ Values of the high-resolution models as retrieved from PDB entry 4F7I for the closed crystal structure of the Tt-IPMDH-Mn ${ }^{2+}-\mathrm{IPM}^{-\mathrm{NADH}}$ quaternary complex and PDB entry $2 \mathrm{Y} 3 \mathrm{Z}$ for the open crystal structure of apo Tt-IPMDH. ${ }^{d}$ The minimum values of discrepancy (in bold) indicate the best correlation between SAXS data and crystallographic model. ${ }^{e}$ The fits for open/closed mixture were obtained with OLIGOMER. ${ }^{f} V_{\text {open }}$ and $V_{\text {closed }}$ correspond to the volume fractions of each state found by OLIGOMER. ${ }^{g}$ The small variations in the calculated molecular mass are due to different numbers of residues resolved in different crystal structures.

654 which is closer to $\mathrm{K}^{185^{\prime}}$, is replaced with Ala, the basicity of $655 \mathrm{~K}^{185^{\prime}}$ is expected to drop dramatically because of the loss of 656 significant electrostatic interactions stabilizing the $\mathrm{I}_{\text {hydride }}$ state, 657 and the decreased basicity will lead to a significant increase in 658 the activation energy of the proton transfer reaction (as shown 659 by the calculations) and decrease the reactivity of the enzyme 660 (as witnessed by the experimental data).

661 In the case of the D245A mutant, the intermediate state 662 seems to be more stable than in the WT enzyme, while the TS2 663 and $\mathrm{P}_{\text {hydride }}$ states are slightly destabilized. As a consequence, 664 the activation energy of the rate-determining hydride transfer 665 step is considerably larger than in the case of the WT, in 666 accordance with the experimentally observed reduced activity 667 [10.9\% (cf. Table 1)] of the D245A mutant. One of the 668 possible roles of the D245 residue might be hindering an overly 669 strong stabilization of the $\mathrm{I}_{\text {hydride }}$ state, thereby decreasing the 670 energy of activation of the hydride transfer step.

671 The calculated relative energies of the N102A mutant are 672 almost identical to those obtained for the WT enzyme. This 673 implies that residue N102 does not contribute significantly to 674 the electrostatic catalysis in IPMDH, which is in accordance 675 with the fact that this mutant retained the highest activity $676[13.9 \%$ (cf. Table 1)] among those of the studied mutants, and 677 the decrease in activity is due most likely to the role of the $678 \mathrm{~N} 102$ side chain in stabilizing the closed conformation, as 679 demonstrated below by the SAXS measurements (cf. Table 2). 680 The QM/MM calculations do not explain the seriously 681 impaired activity [2.7\% (cf. Table 1)] of the Y139A mutant. 682 According to the calculations, the proton and hydride transfer 683 steps in the enzyme should be as facile in this mutant as in the $684 \mathrm{WT}$, in contrast to the experimentally observed 50-fold 685 decrease in reactivity. However, the calculations capture only 686 the electrostatic effect of the amino acid residues in the proton 687 and hydride transfer step of the reaction (cf. Materials and 688 Methods); they do not account for hindrance of the 689 decarboxylation step or for changes in the tertiary structure. 690 In the case of the Y139A mutant, both factors might be 691 relevant, as tyrosine requires a space considerably larger than 692 alanine does, which could lead to some changes in the active 693 site architecture. However, a more plausible scenario is that
Y139 acts as the proton source in the enol-keto tautomeriza- 694 tion process in the decarboxylation step of the reaction as 695 suggested for similar enzymes. ${ }^{1}$ When it is not present, the 696 decarboxylation step becomes rate-limiting, leading to 697 decreased activity, but this effect cannot be reproduced by 698 the calculations. This hypothesis is supported by the fact that 699 the $\mathrm{pK}$ profile of the Y139A mutant is distinctly different from 700 those of the wild type and other mutants, whose $\mathrm{pK}$ profiles 701 follow a simple ionization curve but are shifted compared to 702 each other, suggesting that these systems have the same $\mathrm{pH}-703$ dependent rate-limiting step. The fact that the $\mathrm{pK}$ profile of 704 Y139A is different may indicate that in this mutant the step that 705 follows the hydride transfer (possibly the decarboxylation) 706 becomes the rate-limiting one.

707

On the basis of the computational and experimental results, 708 we suggest the catalytic cycle shown in Scheme 1 . In the first 709 step of the reaction, a proton is abstracted by $\mathrm{K} 185^{\prime}$ from the 710 hydroxyl group of IPM, which is followed by the hydride 711 transfer step. These two processes are strongly assisted by the 712 D241 and D217' residues, with D241 being even more 713 influential. In the decarboxylation step, Y139 protonates C3 of 714 the enol to form the final product, i.e., the keto form. The 715 resting state of the enzyme might be regained by the 716 reprotonation of Y139 by K185', although other protonation 717 pathways may exist for restoring the resting state of the 718 enzyme.

Effects of the Active Site Mutation on the FRET 720 Spectrum of $T t$-IPMDH. Characteristic changes in the 721 fluorescence emission spectrum of the complex of Tt-IPMDH 722 and NADH were observed because of the occurrence of FRET 723 from the Trp side chain(s) of IPMDH to the bound NADH. ${ }^{10}{ }_{724}$ This phenomenon, however, occurs only when the enzyme is 725 complexed with its substrate, the metal ion complex of IPM 726 that stabilizes the closed (active) conformational state of the 727 enzyme; ${ }^{14}$ therefore, FRET is thought to be a characteristic for 728 the domain-closed conformation. This was supported by our 729 previous SAXS measurements, ${ }^{37}$ although an exceptional case 730 was also discovered. ${ }^{57}$

Because the native gel electrophoresis experiment and the 732 near-UV CD spectral changes observed upon the present 733 
734 mutations (cf. above) raised the possibilities of conformational 735 changes upon mutations, we tested formation of the FRET with 736 all the active site mutants of IPMDH mentioned above. Figure 7376 illustrates the FRET spectra of each active site mutant and of

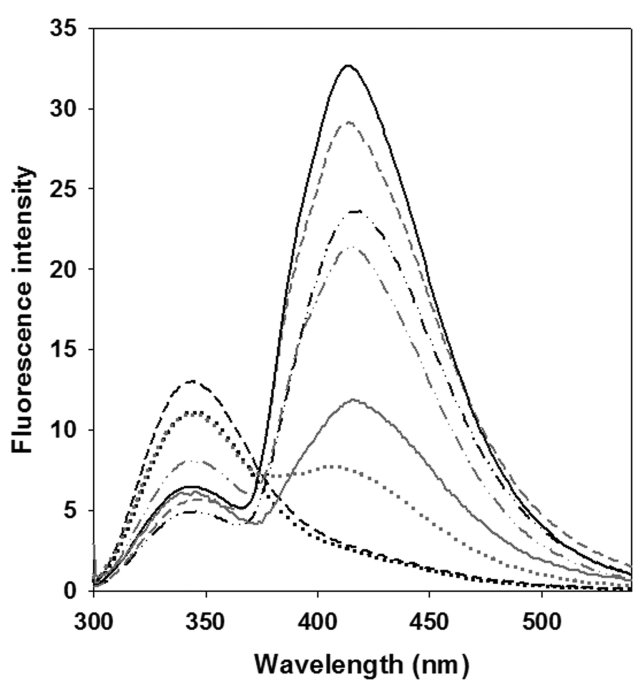

Figure 6. FRET spectra of the investigated active site mutants of TtIPMDH in comparison to that of the wild-type enzyme. FRET experiments with the wild type and various single mutants of IPMDH were conducted as described in Materials and Methods. The FRET spectra are illustrated by using the same types of lines as in the case of CD spectra in Figure 1B. As a comparison, FRET spectra of the previously investigated E270A mutant ${ }^{57}$ (gray dotted line) and N102A (gray dash-dot-dot line) are also illustrated. the previously investigated mutant E270A, compared to those 738 of the wild-type enzyme. Each mutation led to a reduction in 739 the FRET spectrum, but to various extents, fluorescence 740 intensities decreased in the following order: wild type > Y139A 741 $>\mathrm{D} 245 \mathrm{~A} \cong \mathrm{N} 102 \mathrm{~A}>\mathrm{K} 185 \mathrm{~A}>\mathrm{E} 270 \mathrm{~A}>\mathrm{D} 217 \mathrm{~A}=\mathrm{D} 241 \mathrm{~A} .742$ The complete absence of FRET spectra could be observed only 743 in cases of the last two Asp mutants (D241 and D217'). These 744 two aspartates are interacting directly with both the catalytic 745 $\mathrm{Mn}^{2+}$ and the catalytic side-chain K185' (cf. Figure 2). 746

Domain Closure of Tt-IPMDH Is Prevented upon 747 Mutation of the Active Site Residues: SAXS Measure- 748 ments. To test whether domain closure is restricted or 749 prevented in the cases of the mutants exhibiting the partial or 750 complete absence of FRET spectra (cf. above), SAXS 751 measurements have been taken. SAXS is the most appropriate 752 method for testing protein conformational changes occurring in 753 solution if they are accompanied by changes in the shape of the 754 molecule, such as domain closure. The occurrence of domain 755 closure was detected by SAXS in our previous studies with wild- 756 type $T t$-IPMDH in the complex with $\mathrm{Mn}^{2+}-\mathrm{IPM}^{37}$ this 757 experiment was repeated for all the mutants mentioned 758 above. Table 2 summarizes the calculated $R_{\mathrm{g}}$ values as well as 759 the extent of domain closure as expressed by the percentage of 760 the open and closed forms estimated in the manner described 761 in Materials and Methods. The results clearly show that, in 762 addition to the previously demonstrated role of E270, the side 763 chains of $\mathrm{K}^{185^{\prime}}$, D217', D241, and N102 make contributions to 764 the formation of the active domain-closed conformation. The 765 structural basis of these effects is illustrated below (cf. next 766 section). The results especially emphasize the importance of 767 $\mathrm{N} 102$ in domain closure, in spite of the relatively small 768

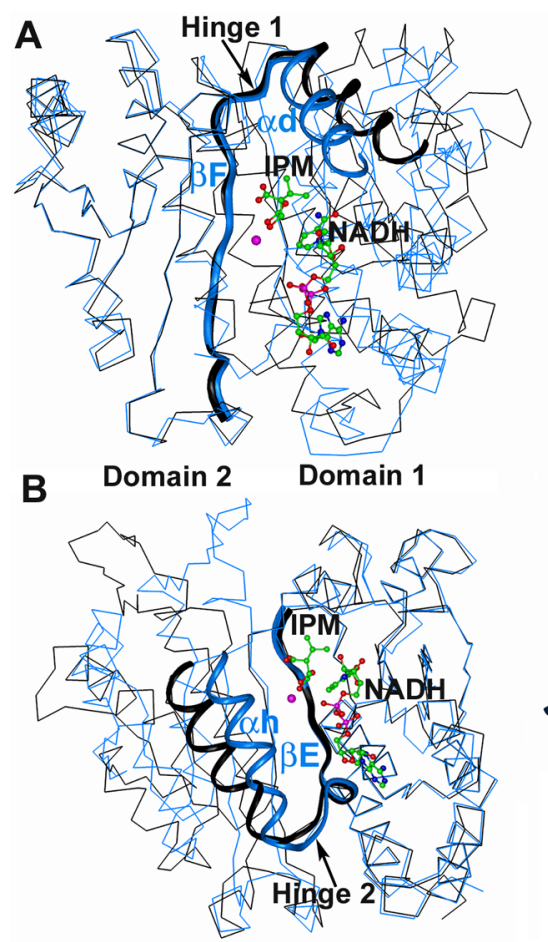

C

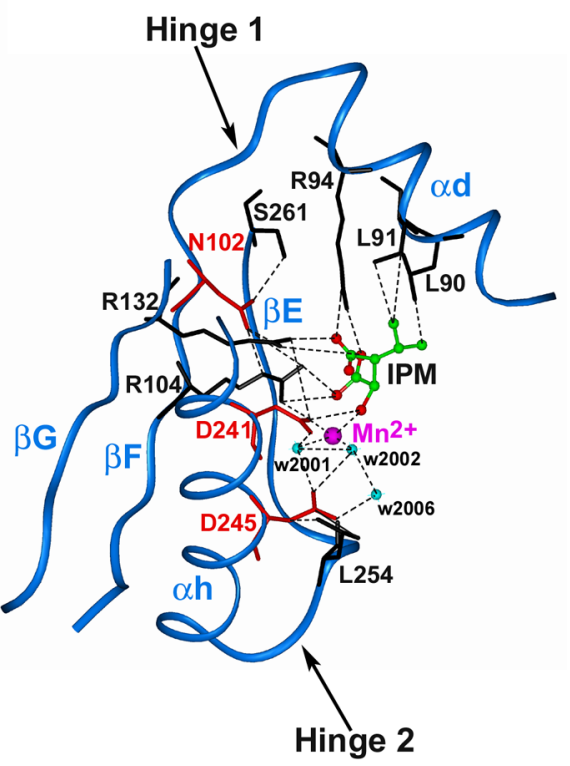

Figure 7. Illustration of the important hinges in the structure of Tt-IPMDH. The positions of (A) hinge 1 and (B) hinge 2 are shown in the whole subunit ( $\mathrm{C} \alpha$ traces) by superimposing $\beta$-sheets $\mathrm{F}$ and $\mathrm{E}$, respectively, of the open (black) and closed (blue) structures. The bound substrates are illustrated as ball and stick models, colored according to atom type. (C) Details of atomic interactions around both hinges are illustrated by dashed lines. The mutated side chains are labeled in red. This figure was prepared by using the atomic coordinates of PDB entry 4F7I of the wild-type quaternary complex of $\mathrm{Tt}$-IPMDH. ${ }^{13}$ 
769 calculated increase in the activation energy of proton transfer 770 (Figure 5A) upon its mutation to alanine. In fact, the N102A 771 mutant exhibits definitely reduced enzyme activity (cf. Table 1). 772 The good correlation between the data from SAXS and 773 FRET measurements indicates that for the mutants exhibiting 774 no or largely reduced FRET spectra domain closure ability is 775 also impaired. Our FRET and SAXS experiments with the 776 various active site mutants have revealed that the easily 777 detectable effects of the bound substrates (mainly $\mathrm{Mn}^{2+}$-IPM) 778 in stabilizing the active domain-closed conformation of the 779 wild-type enzyme are largely prevented upon selective mutation 780 of the presently investigated catalytically important active site 781 side chains.

782 Molecular Graphical Analysis Enlightens the Role of 783 the Mutated Active Site Residues in Domain Closure. 784 Previously, two main hinge regions were identified from 785 comparison of the substrate-free and IPM-bound structures of $786 \mathrm{Tt}$-IPMDH. ${ }^{14}$ The locations of these hinges, namely, hinge 1 787 between $\alpha \mathrm{d}$ and $\beta \mathrm{F}$ and hinge 2 between $\alpha \mathrm{h}$ and $\beta \mathrm{E}$, are 788 illustrated in panels A and B of Figure 7, where the structures of 789 the completely closed unproductive $\mathrm{Tt}$-IPMDH$-\mathrm{Mn}^{2+}-\mathrm{IPM}-$ $790 \mathrm{NADH}$ quaternary complex ${ }^{13}$ and of the completely open 791 apoenzyme $^{14}$ are superimposed. As noted earlier, binding of 792 IPM itself facilitates the relative movement of $\alpha \mathrm{d}$ toward $\beta \mathrm{F}$ via 793 interacting side chains (L90, L91, and R94 from $\alpha$ d and R104 794 from $\beta \mathrm{F}$ ), which is basically the operation of hinge 1 (Figure $7957 \mathrm{C})$. In addition, by interacting with D241 and D245 $(\alpha \mathrm{h})$, 796 IPM mediates the movement of $\alpha$ h relative to central parallel $\beta$ 797 strands $\mathrm{E}-\mathrm{G}$ in the interdomain region, including operation of 798 hinge 2 (cf. Figure 7C). This latter conformational change is 799 also aided by transmission of the conformational signal through 800 the side chains $\mathrm{R} 132(\beta \mathrm{G}) \rightarrow \mathrm{N} 102(\beta \mathrm{F}) \rightarrow \mathrm{S} 261(\beta \mathrm{E})$. 801 Moreover, $\mathrm{D} 241(\alpha \mathrm{h})$ interacts with $\mathrm{R} 132(\beta \mathrm{G})$ as well as $802 \mathrm{D} 245(\alpha \mathrm{h})$ interacts with $\mathrm{L} 254(\beta \mathrm{E})$. Most of these interactions 803 are partially present in the $\mathrm{Tt}$-IPMDH$-\mathrm{Mn}^{2+}-\mathrm{IPM}$ ternary 804 complex $^{14}$ but are complete only in the Tt-IPMDH- $\mathrm{Mn}^{2+}-$ 805 IPM-NADH quaternary complex ${ }^{13}$ when these interactions 806 stabilize the closed states of both hinges 1 and 2 .

807 The third investigated Asp of the active site, D217', resides 808 on helix $\alpha \mathrm{g}^{\prime}$ of the other subunit (Figure 8). Interaction of IPM 809 with this residue along with $\mathrm{D} 241(\alpha \mathrm{h})$ can contribute to 810 stabilization of subunit interactions. As pointed out previously, 811 the subunit-subunit interactions, operating within the dimer, 812 result in further stabilization of the domain-closed conforma813 tion, and domain closure is completed only in the dimeric 814 form. ${ }^{14}$ The other catalytic side chain, $\mathrm{K} 185^{\prime}$, is also located in 815 the other subunit in a loop between $\alpha \mathrm{f}^{\prime}$ and $\beta \mathrm{I}^{\prime}$, and by being 816 inserted into the active site, it may stabilize the subunit817 subunit interactions and thereby the domain-closed form, too. 818 That probably occurs through its simultaneous electrostatic 819 interactions with both D241 and D217' and through its 820 interactions with Y139 (cf. Figure 8B).

821 The other investigated residue, Y139, has been observed to 822 undergo a simultaneous shift and rotation of its ring during 823 domain closure. ${ }^{14}$ It occupies a position on a loop preceding $\beta \mathrm{K}$ 824 and $\beta \mathrm{L}$, i.e., the constituents of the interacting arms of the 825 subunits. In the domain-closed form, the side chain of Y139 is 826 interacting simultaneously with active site residues $\mathrm{R} 132(\beta \mathrm{G})$ 827 and D241 $(\alpha \mathrm{h})$ (cf. Figure 2). Thereby, it can contribute 828 (although less effectively than the active site Asp residues) to 829 stabilization of the domain-closed form and to interdomain 830 communication.
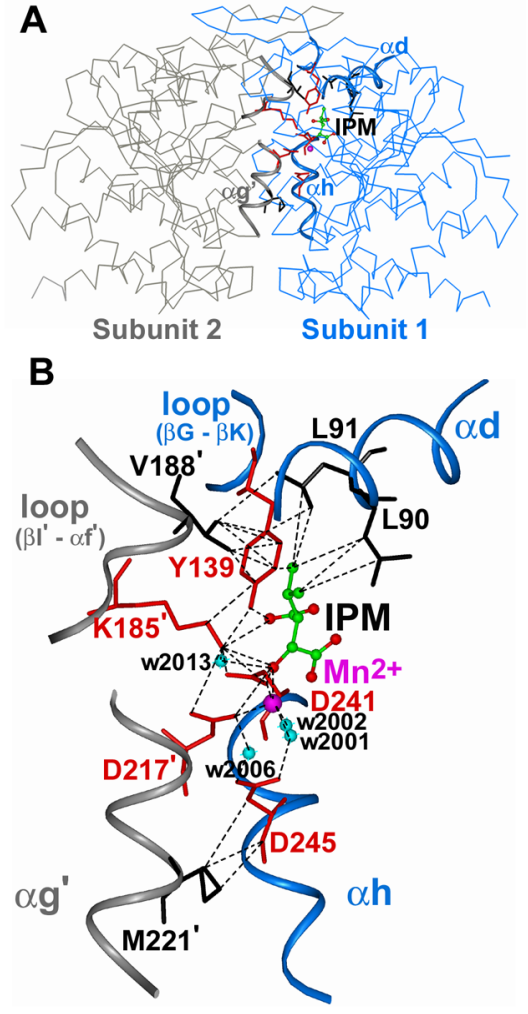

Figure 8. Illustration of the dimeric structure (A) and some of the atomic details of subunit interactions of Tt-IPMDH (B). The interaction of the two subunits ( $\mathrm{C} \alpha$ traces) of IPMDH is shown in panel A. The contacting secondary structural elements are illustrated as ribbons. The details of the latter ones, including the bound substrate, are enlarged in panel B. The mutated side chains are labeled in red. This figure was prepared by using the atomic coordinates of PDB entry 4F7I of the wild-type quaternary complex of Tt-IPMDH. ${ }^{13}$

The side chain of N102 discussed above, which is not a direct 831 substrate binding residue, seems to be more important in the 832 transmission of the conformational signal between the domains, 833 as suggested previously ${ }^{14}$ and illustrated in Figure 7C. Indeed, 834 the side chain of N102 contributes to stabilization of the closed 835 conformation as demonstrated by the SAXS data (Table 2). 836

In summary, the structural analysis provides insight into the 837 possible roles of the investigated active site side chains in 838 domain closure. These mechanistic suggestions are supported 839 by the results of the physicochemical investigations presented 840 above, such as FRET and SAXS.

\section{CONCLUSIONS}

842

The enzyme kinetics and the various physicochemical experi- 843 ments with the investigated point mutants of the active site of 844 Tt-IPMDH led to the identification of the catalytic residues. 845 Our thorough experimental studies (including enzyme kinetics 846 and physicochemical investigations), complemented by molec- 847 ular dynamics simulations and QM/MM calculations, have 848 suggested that the side chains of $\mathrm{K} 185^{\prime}, \mathrm{D} 241, \mathrm{D} 217^{\prime}$, and 849 Y139 significantly contribute to IPMDH catalysis. Thus, the 850 mechanism seemingly fulfills the criteria formulated previously 851 for other $\beta$-hydroxy acid oxidative decarboxylases. Furthermore, 852 by analogy of their mechanisms, these results with IPMDH 853 cleared up the previous uncertainties concerning identification 854 of the catalytic residues in the case of isocitrate dehydrogenase. 855 
856 In addition, IPMDH as a dimer represents a unique example 857 of the functionally related domain motions, in which the main 858 catalytic residues are simultaneously responsible for both 859 strengthening the subunit interactions and operation of the 860 main hinges. Until now, there have been only a few studies in 861 the literature about oligomeric proteins that indicate the 862 possible relationship among domain motions, subunit-subunit 863 interactions, and the proper steric arrangement of the active site 864 residues. $^{58-61}$

\section{${ }_{865}$ ASSOCIATED CONTENT}

\section{S Supporting Information}

867 The Supporting Information is available free of charge on the 868 ACS Publications website at DOI: 10.1021/acs.bio869 chem.5b00839.

870 Details about the methodology of the applied MD 871 simulation as well as about the estimated $\mathrm{p} K_{\mathrm{a}}$ values of 872 the active site Lys residues in the enzyme-substrate 873 complexes of various oxidative decarboxylases (PDF)

\section{AUTHOR INFORMATION}

\section{Corresponding Authors}

876 *Department of Inorganic and Analytical Chemistry, Budapest 877 University of Technology and Economics, Gellért tér 4., H8781111 Budapest, Hungary. Phone: +36 14631 286. E-mail: 879 julianna.olah@mail.bme.hu.

880 *Institute of Enzymology, Research Centre for Natural 881 Sciences, Hungarian Academy of Sciences, Magyar tudósok 882 krt. 2., H-1117 Budapest, Hungary. Phone: +36 13826 773. E883 mail: vas.maria@ttk.mta.hu.

\section{Present Address}

\# P.V.K.: Laboratory of reflectometry and small-angle scattering, 886 Institute of Crystallography RAS, Leninsky pr. 59, 119333 887 Moscow, Russia.

\section{Author Contributions}

889 É.G. and T.S. contributed equally to this work.

\section{Funding}

891 Grant OTKA (NK 108642) of the Hungarian National 892 Research Fund supported this work. Synchrotron facilities of 893 EMBL (Hamburg Outstation) at the beamline at the DORIS 894 storage ring, DESY, were used and supported by the funding 895 from the European Community's Seventh Framework Pro896 gramme (FP7/2007-2013) under Grant Agreement No. 897226716 and from the German Ministry of Education and 898 Science (BMBF) project BIOSCAT, Grant 05K20912. J.O. was 899 supported by the Bolyai János Research Scholarship, and A.L. 900 was supported by the Richter Gedeon Talentum Foundation 901 fellowship.

902 Notes

903 The authors declare no competing financial interest.

\section{$904 \square$ ABBREVIATIONS}

905 IPMDH, 3-isopropylmalate dehydrogenase (EC 1.1.1.85); Tt, 906 T. thermophilus; IPM, (2R,3S)-3-isopropylmalate; FRET, 907 Förster resonance energy transfer; HEPES, 4-(2-hydroxyethyl)908 piperazine-1-ethanesulfonic acid; MES, 2-( $N$-morpholino)909 ethanesulfonic acid; MOPS, 3-( $N$-morpholino)propanesulfonic 910 acid; SAXS, small-angle X-ray scattering; SDS-PAGE, sodium 911 dodecyl sulfate-polyacrylamide gel electrophoresis.

\section{REFERENCES}

912

(1) Aktas, D. F., and Cook, P. F. (2009) A lysine-tyrosine pair carries 913 out acid-base chemistry in the metal ion-dependent pyridine 914 dinucleotide-linked beta-hydroxyacid oxidative decarboxylases. Bio- 915 chemistry 48, 3565-3577.

916

(2) Tao, X., Yang, Z., and Tong, L. (2003) Crystal structures of 917 substrate complexes of malic enzyme and insights into the catalytic 918 mechanism. Structure 11, 1141-1150.

919

(3) Malik, R., and Viola, R. E. (2010) Structural characterization of 920 tartrate dehydrogenase: a versatile enzyme catalyzing multiple 921 reactions. Acta Crystallogr., Sect. D: Biol. Crystallogr. 66, 673-684. 922

(4) Goncalves, S., Miller, S. P., Carrondo, M. A., Dean, A. M., and 923 Matias, P. M. (2012) Induced fit and the catalytic mechanism of 924 isocitrate dehydrogenase. Biochemistry 51, 7098-7115.

925

(5) Grodsky, N. B., Soundar, S., and Colman, R. F. (2000) Evaluation 926 by site-directed mutagenesis of aspartic acid residues in the metal site 927 of pig heart NADP-dependent isocitrate dehydrogenase. Biochemistry 928 39, 2193-2200.

(6) Kim, T.-K., Lee, P., and Colman, R. F. (2003) Critical role of 930 Lys 212 and Tyr140 in porcine NADP-dependent isocitrate dehydro- 931 genase. J. Biol. Chem. 278, 49323-49331.

(7) Huang, Y. C., Grodsky, N. B., Kim, T. K., and Colman, R. F. 933 (2004) Ligands of the Mn2+ bound to porcine mitochondrial NADP- 934 dependent isocitrate dehydrogenase, as assessed by mutagenesis. 935 Biochemistry 43, 2821-2828.

936

(8) Karsten, W. E., Liu, D., Rao, G. S., Harris, B. G., and Cook, P. F. 937 (2005) A catalytic triad is responsible for acid-base chemistry in the 938 Ascaris suum NAD-malic enzyme. Biochemistry 44, 3626-3635. 939

(9) Lee, M. E., Dyer, D. H., Klein, O. D., Bolduc, J. M., Stoddard, B. 940 L., and Koshland, D. E., Jr. (1995) Mutational analysis of the catalytic 941 residues lysine 230 and tyrosine 160 in the NADP+-dependent 942 isocitrate dehydrogenase from Escherichia coli. Biochemistry 34, 378- 943 384.

(10) Dean, A. M., and Dvorak, L. (1995) The role of glutamate 87 in 945 the kinetic mechanism of Thermus thermophilus isopropylmalate 946 dehydrogenase. Protein Sci. 4, 2156-2167.

(11) Miyazaki, K., Kakinuma, K., Terasawa, H., and Oshima, T. 948 (1993) Kinetic analysis on the substrate specificity of 3-isopropylma- 949 late dehydrogenase. FEBS Lett. 332, 35-36.

(12) Fujita, M., Toyooka, Y., Tamegai, H., Eguchi, T., and Kakinuma, 95 K. (2000) Arg-94 is crucial to the catalysis of 3-isopropylmalate 952 dehyrdogenase from Thermus thermophilus HB8. J. Mol. Catal. B: 953 Enzym. 9, 149-155.

954

(13) Palló, A., Oláh, J., Gráczer, E., Merli, A., Závodszky, P., Weiss, 955 M. S., and Vas, M. (2014) Structural and Energetic Basis of 956 Isopropylmalate Dehydrogenase Enzyme Catalysis. FEBS J. 281, 957 5063-5076.

958

(14) Gráczer, É., Merli, A., Singh, R. K., Karuppasamy, M., 959 Závodszky, P., Weiss, M. S., and Vas, M. (2011) Atomic level 960 description of the domain closure in a dimeric enzyme: Thermus 961 thermophilus 3-isopropylmalate dehydrogenase. Mol. BioSyst. 7, 1646- 962 1659.

(15) Gibbs, A. C. (2014) Elements and modulation of functional 964 dynamics. J. Med. Chem. 57, 7819-7837.

965

(16) Darrouzet, E., Moser, C. C., Dutton, P. L., and Daldal, F. (2001) 966 Large scale domain movement in cytochrome bc(1): a new device for 967 electron transfer in proteins. Trends Biochem. Sci. 26, 445-451. 968

(17) Bienert, R., Zimmermann, B., Rombach-Riegraf, V., and Graber, 969 P. (2011) Time-dependent FRET with single enzymes: domain 970 motions and catalysis in $\mathrm{H}(+)$-ATP synthases. ChemPhysChem 12, 971 510-517.

(18) Yang, W. (2010) Lessons learned from UvrD helicase: 973 mechanism for directional movement. Annu. Rev. Biophys. 39, 367- 974 385.

(19) Shen, T., Tai, K., Henchman, R. H., and McCammon, J. A. 976 (2002) Molecular dynamics of acetylcholinesterase. Acc. Chem. Res. 35, 977 $332-340$. 
979 (20) Masaike, T., Mitome, N., Noji, H., Muneyuki, E., Yasuda, R., 980 Kinosita, K., and Yoshida, M. (2000) Rotation of F(1)-ATPase and the 981 hinge residues of the beta subunit. J. Exp Biol. 203, 1-8.

982 (21) Kannan, N., Neuwald, A. F., and Taylor, S. S. (2008) Analogous 983 regulatory sites within the alphaC-beta4 loop regions of ZAP-70 984 tyrosine kinase and AGC kinases. Biochim. Biophys. Acta, Proteins 985 Proteomics 1784, 27-32.

986 (22) Nakanishi-Matsui, M., Sekiya, M., Nakamoto, R. K., and Futai, 987 M. (2010) The mechanism of rotating proton pumping ATPases. 988 Biochim. Biophys. Acta, Bioenerg. 1797, 1343-1352.

989 (23) Vas, M., Varga, A., and Gráczer, É. (2010) Insight into the 990 mechanism of domain movements and their role in enzyme function: 991 example of 3-phosphoglycerate kinase. Curr. Protein Pept. Sci. 11, 118992147.

993 (24) Palmai, Z., Seifert, C., Grater, F., and Balog, E. (2014) An 994 allosteric signaling pathway of human 3-phosphoglycerate kinase from 995 force distribution analysis. PLoS Comput. Biol. 10, e1003444.

996 (25) Owen, G. R., Stoychev, S., Achilonu, I., and Dirr, H. W. (2014) 997 Phosphorylation- and nucleotide-binding-induced changes to the 998 stability and hydrogen exchange patterns of JNK1betal provide 999 insight into its mechanisms of activation. J. Mol. Biol. 426, 3569-3589. 1000 (26) Yang, Z., Floyd, D. L., Loeber, G., and Tong, L. (2000) 1001 Structure of a closed form of human malic enzyme and implications 1002 for catalytic mechanism. Nat. Struct. Biol. 7, 251-257.

1003 (27) Gerstein, M., and Echols, N. (2004) Exploring the range of 1004 protein flexibility, from a structural proteomics perspective. Curr. Opin. 1005 Chem. Biol. 8, 14-19.

1006 (28) Bahar, I., Chennubhotla, C., and Tobi, D. (2007) Intrinsic 1007 dynamics of enzymes in the unbound state and relation to allosteric 1008 regulation. Curr. Opin. Struct. Biol. 17, 633-640.

1009 (29) Henzler-Wildman, K. A., Lei, M., Thai, V., Kerns, S. J., Karplus, 1010 M., and Kern, D. (2007) A hierarchy of timescales in protein dynamics 1011 is linked to enzyme catalysis. Nature 450, 913-916.

1012 (30) Gráczer, É., Varga, A., Melnik, B., Semisotnov, G., Závodszky, P., 1013 and Vas, M. (2009) Symmetrical refolding of protein domains and 1014 subunits: example of the dimeric two-domain 3-isopropylmalate 1015 dehydrogenases. Biochemistry 48, 1123-1134.

1016 (31) Laemmli, U. K. (1970) Cleavage of structural proteins during 1017 the assembly of the head of bacteriophage T4. Nature 227, 680-685. 1018 (32) Ornstein, L. (1964) Disc Electrophoresis. I. Background and 1019 Theory. Ann. N.Y. Acad. Sci. 121, 321-349.

1020 (33) Konarev, P. V., Volkov, V. V., Sokolova, A. V., Koch, M. H. J., 1021 and Svergun, D. I. (2003) PRIMUS: a Windows PC-based system for 1022 small-angle scattering data analysis. J. Appl. Crystallogr. 36, 1277-1282. 1023 (34) Guinier, A. (1939) La diffraction des rayons X aux trés petits 1024 angles; application á l'étude de phénoménes ultramicroscopiques. Ann. 1025 Phys. 12, 166-237.

1026 (35) Svergun, D. I. (1992) Determination of the regularisation 1027 parameter in indirect-transform methods using perceptual criteria. $J$. 1028 Appl. Crystallogr. 25, 495-503.

1029 (36) Svergun, D. I., Barberato, C., and Koch, M. H. J. (1995) 1030 CRYSOL - a program to evaluate X-ray solution scattering of 1031 biological macromolecules from atomic coordinates. J. Appl. 1032 Crystallogr. 28, 768-773.

1033 (37) Gráczer, É., Konarev, P. V., Szimler, T., Bacsó, A., Bodonyi, A., 1034 Svergun, D. I., Závodszky, P., and Vas, M. (2011) Essential role of the 1035 metal-ion in the IPM-assisted domain closure of 3-isopropylmalate 1036 dehydrogenase. FEBS Lett. 585, 3297-3302.

1037 (38) Brooks, B. R., Brooks, C. L., 3rd, Mackerell, A. D., Jr., Nilsson, 1038 L., Petrella, R. J., Roux, B., Won, Y., Archontis, G., Bartels, C., Boresch, 1039 S., Caflisch, A., Caves, L., Cui, Q., Dinner, A. R., Feig, M., Fischer, S., 1040 Gao, J., Hodoscek, M., Im, W., Kuczera, K., Lazaridis, T., Ma, J., 1041 Ovchinnikov, V., Paci, E., Pastor, R. W., Post, C. B., Pu, J. Z., Schaefer, 1042 M., Tidor, B., Venable, R. M., Woodcock, H. L., Wu, X., Yang, W., 1043 York, D. M., and Karplus, M. (2009) CHARMM: the biomolecular 1044 simulation program. J. Comput. Chem. 30, 1545-1614.

1045 (39) Bash, P. A., Field, M. J., Davenport, R. C., Petsko, G. A., Ringe, 1046 D., and Karplus, M. (1991) Computer simulation and analysis of the reaction pathway of triosephosphate isomerase. Biochemistry 30, 1047 $5826-5832$.

1048

(40) Tian, B. X., and Eriksson, L. A. (2011) Catalytic mechanism and 1049 roles of Arg197 and Thr183 in the Staphylococcus aureus sortase A 1050 enzyme. J. Phys. Chem. B 115, 13003-13011.

1051

(41) Liao, R. Z., and Thiel, W. (2013) Convergence in the QM-only 1052 and $\mathrm{QM} / \mathrm{MM}$ modeling of enzymatic reactions: A case study for 1053 acetylene hydratase. J. Comput. Chem. 34, 2389-2397.

1054

(42) Perez-Gallegos, A., Garcia-Viloca, M., Gonzalez-Lafont, A., and 1055 Lluch, J. M. (2014) A QM/MM study of the associative mechanism 1056 for the phosphorylation reaction catalyzed by protein kinase A and its 1057 D166A mutant. J. Comput.-Aided Mol. Des. 28, 1077-1091.

1058

(43) Garcia-Viloca, M., Truhlar, D. G., and Gao, J. (2003) Reaction- 1059 path energetics and kinetics of the hydride transfer reaction catalyzed 1060 by dihydrofolate reductase. Biochemistry 42, 13558-13575. 1061

(44) Szarek, P., Dyguda-Kazimierowicz, E., Tachibana, A., and 1062 Sokalski, W. A. (2008) Physical nature of intermolecular interactions 1063 within cAMP-dependent protein kinase active site: differential 1064 transition state stabilization in phosphoryl transfer reaction. J. Phys. 1065 Chem. B 112, 11819-11826.

1066

(45) Cheng, Y., Zhang, Y., and McCammon, J. A. (2005) How does 1067 the cAMP-dependent protein kinase catalyze the phosphorylation 1068 reaction: an ab initio QM/MM study. J. Am. Chem. Soc. 127, 1553- 1069 1562.

1070

(46) Warshel, A., Sharma, P. K., Kato, M., Xiang, Y., Liu, H., and 1071 Olsson, M. H. (2006) Electrostatic basis for enzyme catalysis. Chem. 1072 Rev. 106, 3210-3235.

1073

(47) Harvey, J. N. (2004) Spin-forbidden CO ligand recombination 1074 in myoglobin. Faraday Discuss. 127, 165-177.

(48) Frisch, M. J., Trucks, G. W., Schlegel, H. B., Scuseria, G. E., 1076 Robb, M. A., Cheeseman, J. R., Scalmani, G., Barone, V., Mennucci, B., 1077 Petersson, G. A., Nakatsuji, H., Caricato, M., Li, X., Hratchian, H. P., 1078 Izmaylov, A. F., Bloino, J., Zheng, G., Sonnenberg, J. L., Hada, M., 1079 Ehara, M., Toyota, K., Fukuda, R., Hasegawa, J., Ishida, M., Nakajima, 1080 T., Honda, Y., Kitao, O., Nakai, H., Vreven, T., Montgomery, J. A., Jr., 1081 Peralta, J. E., Ogliaro, F., Bearpark, M., Heyd, J. J., Brothers, E., Kudin, 1082 K. N., Staroverov, V. N., Kobayashi, R., Normand, J., Raghavachari, K., 1083 Rendell, A., Burant, J. C., Iyengar, S. S., Tomasi, J., Cossi, M., Rega, N., 1084 Millam, N. J., Klene, M., Knox, J. E., Cross, J. B., Bakken, V., Adamo, 1085 C., Jaramillo, J., Gomperts, R., Stratmann, R. E., Yazyev, O., Austin, A. 1086 J., Cammi, R., Pomelli, C., Ochterski, J. W., Martin, R. L., Morokuma, 1087 K., Zakrzewski, V. G., Voth, G. A., Salvador, P., Dannenberg, J. J., 1088 Dapprich, S., Daniels, A. D., Farkas, Ö., Foresman, J. B., Ortiz, J. V., 1089 Cioslowski, J., and Fox, D. J. (2009) Gaussian 09, revision B.01, 1090 Gaussian, Inc., Wallingford, CT.

(49) Page, H. Tinker: Software tools for molecular design (http:// 1092 dasher.wustl.edu/tinker/) (accessed 5 October, 2011). 1093

(50) Olsson, M. H., Søndergaard, C. R., Rostkowski, M., and Jensen, 1094 J. H. (2011) PROPKA3: Consistent Treatment of Internal and Surface 1095 Residues in Empirical pKa predictions. J. Chem. Theory Comput. 7, 1096 525-537.

(51) Søndergaard, C. R., Olsson, M. H., Rostkowski, M., and Jensen, 1098 J. H. (2011) Improved Treatment of Ligands and Coupling Effects in 1099 Empirical Calculation and Rationalization of pKa Values. J. Chem. 1100 Theory Comput. 7, 2284-2295.

1101

(52) Miyazaki, K., and Oshima, T. (1993) Tyr-139 in Thermus 1102 thermophilus 3-isopropylmalate dehydrogenase is involved in catalytic 1103 function. FEBS Lett. 332, 37-38.

1104

(53) Gráczer, É., Bacsó, A., Kónya, D., Kazi, A., Soós, T., Molnár, L., 1105 Szimler, T., Beinrohr, L., Szilágyi, A., Závodszky, P., and Vas, M. 1106 (2014) Drugs Against Mycobacterium Tuberculosis 3-Isopropylmalate 1107 Dehydrogenase Can be Developed using Homologous Enzymes as 1108 Surrogate Targets. Protein Pept Lett. 21, 1295-1307. 1109

(54) Berdis, A. J., and Cook, P. F. (1993) Chemical mechanism of 6- 1110 phosphogluconate dehydrogenase from Candida utilis from $\mathrm{pH} 1111$ studies. Biochemistry 32, 2041-2046.

1112

(55) Price, N. E., and Cook, P. F. (1996) Kinetic and chemical 1113 mechanisms of the sheep liver 6-phosphogluconate dehydrogenase. 1114 Arch. Biochem. Biophys. 336, 215-223. 
1116 (56) Quartararo, C. E., Hazra, S., Hadi, T., and Blanchard, J. S. 1117 (2013) Structural, Kinetic and Chemical Mechanism of Isocitrate 1118 Dehydrogenase-1 from Mycobacterium tuberculosis. Biochemistry 52, 1119 1765-1775.

1120 (57) Gráczer, É., Palló, A., Oláh, J., Szimler, T., Konarev, P. V., 1121 Svergun, D. I., Merli, A., Závodszky, P., Weiss, M. S., and Vas, M. 1122 (2015) Glutamate 270 plays an essential role in $\mathrm{K}(+)$-activation and 1123 domain closure of Thermus thermophilus isopropylmalate dehydro1124 genase. FEBS Lett. 589, 240-245.

1125 (58) Hayward, S., and Kitao, A. (2006) Molecular dynamics 1126 simulations of NAD+-induced domain closure in horse liver alcohol 1127 dehydrogenase. Biophys. J. 91, 1823-1831.

1128 (59) Dey, S., Hu, Z., Xu, X. L., Sacchettini, J. C., and Grant, G. A. 1129 (2007) The effect of hinge mutations on effector binding and domain 1130 rotation in Escherichia coli D-3-phosphoglycerate dehydrogenase. J. 1131 Biol. Chem. 282, 18418-18426.

1132 (60) Cunningham, C. N., Krukenberg, K. A., and Agard, D. A. (2008) 1133 Intra- and intermonomer interactions are required to synergistically 1134 facilitate ATP hydrolysis in Hsp90. J. Biol. Chem. 283, 21170-21178. 1135 (61) Pemberton, T. A., Srivastava, D., Sanyal, N., Henzl, M. T., 1136 Becker, D. F., and Tanner, J. J. (2014) Structural studies of yeast 1137 Delta(1)-pyrroline-5-carboxylate dehydrogenase (ALDH4A1): active 1138 site flexibility and oligomeric state. Biochemistry 53, 1350-1359.

1139 (62) Gráczer, É., Lionne, C., Závodszky, P., Chaloin, L., and Vas, M. 1140 (2013) Transient kinetic studies reveal isomerization steps along the 1141 kinetic pathway of Thermus thermophilus 3-isopropylmalate dehy1142 drogenase. FEBS J. 280, 1764-1772. 